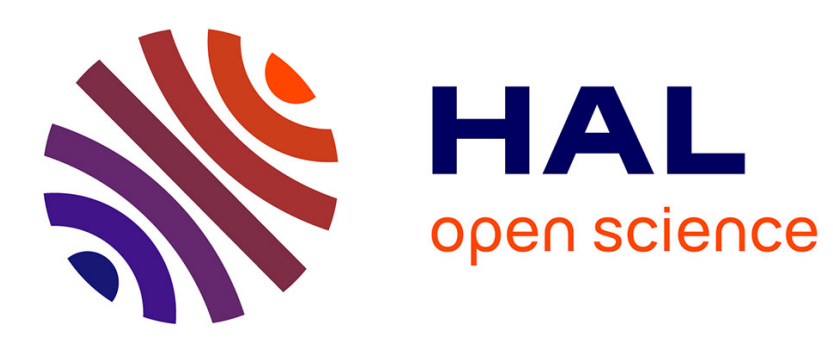

\title{
Error estimate in a finite volume approximation of the partial asymptotic domain decomposition.
}

\author{
Grigory Panasenko, Marie-Claude Viallon
}

\section{To cite this version:}

Grigory Panasenko, Marie-Claude Viallon. Error estimate in a finite volume approximation of the partial asymptotic domain decomposition.. Mathematical Methods in the Applied Sciences, 2013, 36 (14), pp.1892-1917. 10.1002/mma.2735 . hal-00916745

\section{HAL Id: hal-00916745 \\ https://hal.science/hal-00916745}

Submitted on 10 Dec 2013

HAL is a multi-disciplinary open access archive for the deposit and dissemination of scientific research documents, whether they are published or not. The documents may come from teaching and research institutions in France or abroad, or from public or private research centers.
L'archive ouverte pluridisciplinaire HAL, est destinée au dépôt et à la diffusion de documents scientifiques de niveau recherche, publiés ou non, émanant des établissements d'enseignement et de recherche français ou étrangers, des laboratoires publics ou privés. 


\title{
ERROR ESTIMATE IN A FINITE VOLUME APPROXIMATION OF THE PARTIAL ASYMPTOTIC DOMAIN DECOMPOSITION *
}

\author{
GRIGORY PANASENKO ${ }^{\dagger}$ AND MARIF-CI.AUDF VIALLON
}

\begin{abstract}
The method of asymptotic partial domain decomposition (MAPDD) has been proposed for partial differential equations set in rod structures, depending on a small parameter. It reduces the dimension of the problem (or simplifies it in another way) in the main part of the domain keeping the initial formulation in the remaining part and prescribing the asymptotically precise conditions on the interface. This paper is devoted to the finite volume implementation of the MAPDD. We consider a model problem in a thin domain (its thickness is a small parameter). We obtain an error estimate, expressed in terms of the small parameter and the step of the mesh.
\end{abstract}

Key words. energy estimates, homogenization, asymptotic expansion, finite volume scheme, elliptic problem

AMS subject classifications. 35B27, 35Q53, 35C20, 35J25, 65N12, 76M12

1. Introduction. A great number of applied problems contain small parameters. Normally their presence either in the equation or in the domain makes the numerical implementation more complicated, more time and memory consuming. This issue emphasizes the importance of the asymptotic methods studying the behavior of the solution as the small parameter tends to zero. Nevertheless the asymptotic methods are often related to some cumbersome calculations, or they are not too clear for engineers. That is why some special numerical methods taking into account the asymptotic behavior of the solution were developed. One of such ideas has been implemented in the numerical schemes uniform with respect to the small parameter $[2],[7]$ or in some projection numerical methods with a special choice of the projection space basis taking into account the regular part of an asymptotic solution [8],[17], (the idea of projection procedure has been widely used in engineering).

The present paper is devoted to the finite volume implementation of the method of partial asymptotic domain decomposition (MAPDD), introduced in [11],[12],[13]. This method reduces the problem to a simplified form on some subdomain of regular asymptotic behavior of the solution (for example, by means of the dimension reduction) keeping the initial formulation on a small part of the domain where the asymptotic behavior is singular (for example, where the boundary layers are located). Then these two models are coupled by some special interface conditions respecting with great accuracy the asymptotic expansion of the solution. These interface conditions are obtained from some projection procedure in the variational formulation where the projection subspace keeps the asymptotic behavior of the solution out of a boundary layer zone.

In the present paper we consider a two-dimensional setting, although the finite volume implementation of the MAPDD can be generalized for three-dimensional settings. In this case the three dimensional finite volumes should be coupled to the one dimensional finite volume approximations. However, the details are rather technical and it will be published in a separate paper. In section 2, we remind the method of partial asymptotic domain decomposition (MAPDD) for the thin rod structures. The rod structures $[10]$ play an important role in the modelling of the mechanical behavior

\footnotetext{
*Université de Lyon, Institut Camille Jordan, UMR CNRS 5208, Faculté des Sciences et Techniques, 23 rue Docteur Paul Michelon, 42023 Saint-Etienne Cedex 2, France.

$\dagger$ (Grigory.panasenko@univ-st-etienne.fr).
} 
of the industrial installations, in the human or animal blood circulatory system [16]. The dimension reduction in such a modelling is a natural approach [10], [13], [14], [24][40], although the full-dimensional models have to be kept in the neighborhoods of the bifurcations or junctions. So the MAPDD gives the asymptotically exact answer what should be the correct interface conditions.

In section 3, we consider the Poisson equation as model problem. The Poisson equation is set in a thin bidimensional rod structure (union of some thin rectangles [8]). The same approach may be used for more general equations, for example, for the fluid dynamics equation [18]. The MAPDD version for the Poisson equation is a hybrid 1D-2D dimension model, where 1D and 2D problems are coupled (the so called partially decomposed problem).

However, applying the principle of superposition one can decompose completely (and not partially) this hybrid 1D-2D dimension problem and reduce it to some set of independent auxiliary completely 1D subproblems and completely 2D subproblems and to a linear algebraic system of equations. We study the properties of the coefficients and the solution of this system.

In section 4, we obtain the asymptotic expansion of the solutions, and of their first and second derivatives, of the 2D auxiliary subproblems. These results are important for the error estimate at the end of the paper.

In section 5, we discuss the finite volume implementation of the MAPDD set in rod structures. The finite element implementation of the MAPDD has been discussed in [6], and the finite volume implementation in the case of the simplest thin structure, containing only one branch, was considered in $\lceil 15]$. Below we consider a general 2D thin structure containing $n$ branches. The finite volume implementation respects the mesh admissibility condition [19]. This condition allows to follow the classical idea of the proof of the error estimates and convergence. The goal is to study the influence and the contribution of the small parameter $\epsilon$ in the overall error estimate.

The main result obtained in the section 6 , is the global error estimate, combining the influence of both : small parameter $\epsilon$ and the mesh step $h$.

In section 7, we present some numerical results that prove the good behaviour of the hybrid finite volume scheme.

2. The method of asymptotic partial decomposition of domain. The method of partial asymptotic decomposition of domain was proposed in [11],[12] for partial differential equations, stated in rod structures, i.e. in some connected unions of thin cylinders. It is based on the information about the structure of the asymptotic solution in different parts of such domain. The principal idea of the method is to extract the subdomain of singular behavior of the solution and to simplify the problem in the subdomain of regular behavior of the solution. The special interface conditions are imposed on the common boundary of these partially decomposed subdomains.

Consider the Poisson equation set in a rod structure. Describe the method in a simple case of a rod structure containing one bundle.

Let $e_{1}, \ldots, e_{n}$ be $n$ closed segments in $\mathbb{R}^{2}$, which have a single common point $O$ (i.e. the origin of the coordinate system), and let it be the common end point of all these segments. Denote $O_{j}$ the second end of $e_{j}$, i.e., let $e_{j}$ be $O O_{j}$. Define the graph

$$
B=\cup_{j=1}^{n} e_{j} .
$$

Denote $(x, y)$ the coordinates in the canonical base in $\mathbb{R}^{2}$. Define the local coordinates $\left(x^{e_{j}}, y^{e_{j}}\right)$, associated with the segment $e_{j}$. This local system is obtained by the 
rotation around the origin $O$ of the coordinate axes such that the new axe $O x^{e_{j}}$, being the image of $O x$, coincides with the direction $O O_{j}$.

Denote $\varphi_{j}$ the angle between $O x$ and $O x^{e_{j}}, j=1, \ldots, n$. We have $\left[\begin{array}{l}x \\ y\end{array}\right]=\Gamma_{j}\left[\begin{array}{l}x^{e_{j}} \\ y^{e_{j}}\end{array}\right]$, with $\Gamma_{j}=\left[\begin{array}{cc}\cos \left(\varphi_{j}\right) & -\sin \left(\varphi_{j}\right) \\ \sin \left(\varphi_{j}\right) & \cos \left(\varphi_{j}\right)\end{array}\right]$, matrix of the rotation around the center $\mathrm{O}$ of angle $\varphi_{j}, j=1, \ldots, n$.

Denote $B_{j}^{\varepsilon}$ the rectangles

$$
\left\{(x, y) \mid x^{e_{j}} \in\left(0, l_{j}\right), \quad y^{e_{j}} \in\left(-\frac{\varepsilon \theta_{j}}{2}, \frac{\varepsilon \theta_{j}}{2}\right)\right\}
$$

where $l_{j}=\left|e_{j}\right|$ stands for the length of $e_{j}, \theta_{1}, \ldots, \theta_{n}$ are some positive numbers independent of $\varepsilon$.

Let $\omega_{0}, \ldots, \omega_{n}$ be $n+1$ bounded domains in $\mathbb{R}^{2}$, Denote

$$
\omega_{j}^{\varepsilon}=\left\{(x, y) \mid \frac{(x, y)-O_{j}}{\varepsilon} \in \omega_{j}\right\}
$$

by convention, $O_{0}=O$. Denote

$$
\Omega_{\varepsilon}=\cup_{j=1}^{n} B_{j}^{\varepsilon} \cup \cup_{j=0}^{n} \omega_{j}^{\varepsilon} .
$$

Assume that $\Omega_{\varepsilon}$ has a smooth boundary $\partial \Omega_{\varepsilon} \in C^{2}$. Denote the small sides of rectangles $B_{j}^{\varepsilon}$ :

$$
\begin{aligned}
& \beta_{j}^{\varepsilon}=\left\{(x, y) \mid x^{e_{j}}=0, \quad y^{e_{j}} \in\left(-\frac{\varepsilon \theta_{j}}{2}, \frac{\varepsilon \theta_{j}}{2}\right)\right\}, \\
& \hat{\beta}_{j}^{\varepsilon}=\left\{(x, y) \mid x^{e_{j}}=l_{j}, \quad y^{e_{j}} \in\left(-\frac{\varepsilon \theta_{j}}{2}, \frac{\varepsilon \theta_{j}}{2}\right)\right\} .
\end{aligned}
$$

Assume that $\hat{\beta}_{j}^{\varepsilon} \subset \partial \Omega_{\varepsilon}$ (see Fig. 2.1).

Consider the boundary value problem set in the domain $\Omega_{\varepsilon}$ :

$$
\left\{\begin{array}{l}
\triangle u_{\varepsilon}=f, \quad \text { in } \quad \Omega_{\varepsilon} \\
u_{\varepsilon}=0, \quad \text { on } \hat{\beta}_{j}^{\epsilon}, j=1, \ldots, n \\
\frac{\partial u_{\varepsilon}}{\partial n}=0, \quad \text { on } \quad \partial \Omega_{\varepsilon} \backslash\left(\cup_{j=1}^{n} \hat{\beta}_{j}^{\varepsilon}\right)
\end{array}\right.
$$

with the right hand side from $L^{2}\left(\Omega_{\epsilon}\right)$.

Define $f(x, y)=f_{j}\left(x^{e_{j}}\right)$, if $(x, y) \in B_{j}^{\epsilon}, j=1, \ldots, n$, where $f_{j}$ are independent of $\varepsilon$ and vanish in some neighborhood of the ends of the segments $e_{j}$ (and so, the size of these neighborhoods is independent of $\varepsilon$ ).

An asymptotic analysis (see [10], [14]) shows that an asymptotic solution does not depend on $y^{e_{j}}$ at some distance $\delta, \delta>0$ from $O$, i.e. if when $\delta \leq x^{e_{j}} \leq l_{j}-\delta, j=$ $1, \ldots, n$. It allows to apply the method of asymptotic partial decomposition of the domain (MAPDD, see [11], [12],[14]). Let us describe this method for the above 


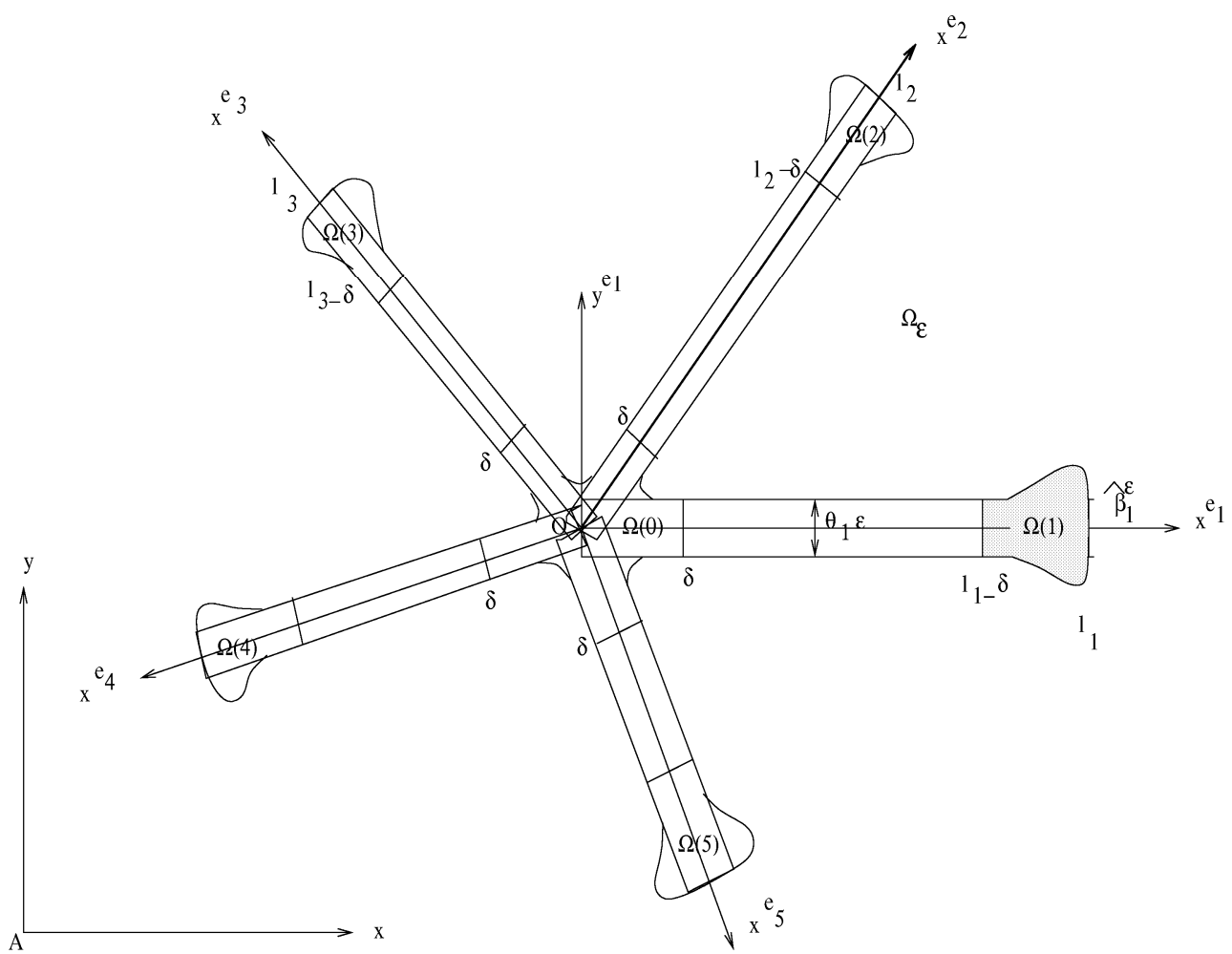

FIG. 2.1. The thin domain $\Omega_{\varepsilon}$

model problem. Let us cut the rectangles $B_{j}^{\varepsilon}$ at the distance $\delta, \delta>>\varepsilon, \delta<<1$, from the ends $O$ and $O_{j}$ of each segment $e_{j}$, i.e., let us trace the segments $\gamma_{j}^{\prime}$ and $\gamma_{j}^{\prime \prime}$ :

$$
\begin{gathered}
\gamma_{j}^{\prime}=\left\{(x, y) \mid x^{e_{j}}=\delta, \quad y^{e_{j}} \in\left(-\frac{\varepsilon \theta_{j}}{2}, \frac{\varepsilon \theta_{j}}{2}\right)\right\}, \\
\gamma_{j}^{\prime \prime}=\left\{(x, y) \mid x^{e_{j}}=l_{j}-\delta, \quad y^{e_{j}} \in\left(-\frac{\varepsilon \theta_{j}}{2}, \frac{\varepsilon \theta_{j}}{2}\right)\right\},
\end{gathered}
$$

and denote $\Omega(j)$ the truncated connected part of $\Omega_{\varepsilon}$, such that its closure contains $O_{j}$. Replace the parts between $\gamma_{j}^{\prime}$ and $\gamma_{j}^{\prime \prime}$ by the parts $e_{0 j}=\left\{(x, y) \mid y^{e_{j}}=0, x^{e_{j}} \in\right.$ $\left.\left(\delta, l_{j}-\delta\right)\right\}$, of the segments $e_{j}$. Remind that $f=0$ on $\Omega(j), j=0, \ldots, n$.

Consider the following 1D-2D hybrid dimension partially decomposed problem 


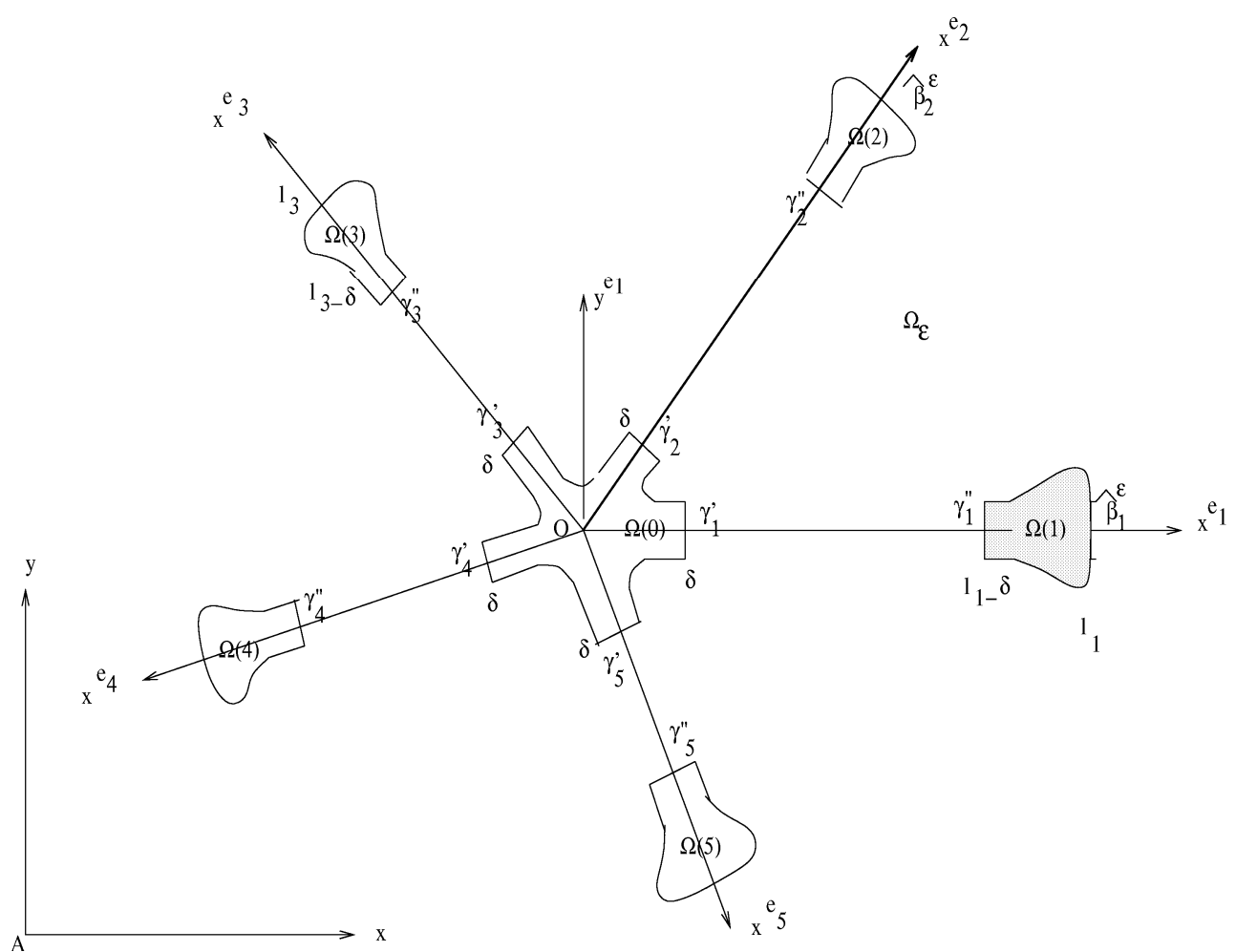

FIG. 2.2. The decomposed domain

$$
\left\{\begin{array}{l}
v_{j}^{\prime \prime}\left(x^{e_{j}}\right)=f_{j}\left(x^{e_{j}}\right), x^{e_{j}} \in\left(\delta, l_{j}-\delta\right), j=1, \ldots, n \\
\triangle u_{j}(x, y)=0,(x, y) \in \Omega(j), j=0, \ldots, n \\
\frac{\partial u_{0}}{\partial n}(x, y)=0,(x, y) \in \partial \Omega(0) \backslash\left(\cup_{j=1}^{n} \gamma_{j}^{\prime}\right) \\
u_{0}(x, y)=v_{j}(\delta),(x, y) \in \gamma_{j}^{\prime}, j=1, \ldots, n \\
u_{j}(x, y)=v_{j}\left(l_{j}-\delta\right),(x, y) \in \gamma_{j}^{\prime \prime}, j=1, \ldots, n \\
\left.u_{j}\right|_{\hat{\beta}_{j}^{\varepsilon}}=0, j=1, \ldots, n \\
\frac{\partial u_{j}}{\partial n}=0, \quad \text { on } \quad \partial \Omega(j) \backslash\left(\gamma_{j}^{\prime \prime} \cup \hat{\beta}_{j}^{\varepsilon}\right), j=1, \ldots, n \\
v_{j}^{\prime}(\delta)=\frac{1}{\theta_{j} \varepsilon} \int_{\gamma_{j}^{\prime}} \frac{\partial u_{0}}{\partial n} d \gamma, j=1, \ldots, n \\
v_{j}^{\prime}\left(l_{j}-\delta\right)=-\frac{1}{\theta_{j} \varepsilon} \int_{\gamma_{j}^{\prime \prime}} \frac{\partial u_{j}}{\partial n} d \gamma, j=1, \ldots, n
\end{array}\right.
$$

Denote $u^{d}(x, y)=\left\{\begin{array}{lll}u_{j}(x, y) & \text { if } \quad(x, y) \in \Omega(j), j=0, \ldots, n \\ v_{j}\left(x^{e_{j}}\right) & \text { if } \quad(x, y) \in B_{j}^{\varepsilon}, x^{e_{j}} \in\left(\delta, l_{j}-\delta\right), j=1, \ldots, n\end{array}\right.$, the solution of the partially decomposed problem. The theory of the method of asymptotic partial decomposition of the domain gives the following estimate for the difference of the exact solution and the solution of the partially decomposed problem [11], [12],[14]: 
for any $K>0$ there exists $\hat{K}$ independent of $\varepsilon$, such that if $\delta=\hat{K} \varepsilon|\ln \varepsilon|$ then

$$
\left\|u_{\varepsilon}-u^{d}\right\|_{H^{1}\left(\Omega_{\varepsilon}\right)}=O\left(\varepsilon^{K}\right) .
$$

Our aim is to obtain a finite volume approximation $u_{\mathcal{T}}^{d}$ for $u^{d}$ and to get the overall estimate for the difference $u_{\varepsilon}-u_{\mathcal{T}}^{d}$. Thanks to the above estimate, it is sufficient to evaluate $\left\|u^{d}-u_{\mathcal{T}}^{d}\right\|$ in a suitable norm. Namely, we will specify the dependence of this estimate on $\varepsilon$ and the mesh step $h$.

\section{Complete decomposition of the partially decomposed problem.}

3.1. Auxiliary problems. In order to solve (2.2), we introduce auxiliary variables $\alpha_{0 j}$ and $\alpha_{j}, \mathrm{j}=1, \ldots, \mathrm{n}$, allowing to solve separately the $1 \mathrm{D}$-problems and the 2D-problems. It means that due to the linearity of the problem (2.2), it can be decomposed completely and not only partially. Indeed, the components of $u^{d}$ can be presented in the following form:

$$
\left\{\begin{array}{l}
v_{j}=\bar{v}_{j}+\alpha_{0 j} \widetilde{v}_{j 0}+\alpha_{j} \widetilde{v}_{j j}, j=1, \ldots, n \\
u_{0}=\sum_{j=1}^{n} \alpha_{0 j} \widetilde{u}_{0 j} \\
u_{j}=\alpha_{j} \widetilde{u}_{j}, j=1, \ldots, n
\end{array}\right.
$$

Here $\bar{v}_{j}, \widetilde{v}_{j 0}, \widetilde{v}_{j j}, \widetilde{u}_{0 j}, \widetilde{u}_{j}, \mathrm{j}=1, \ldots, \mathrm{n}$, are the solutions (respectively) of the following independent problems $\bar{P}_{j}, \widetilde{P}_{j 0}, \widetilde{P}_{j, j}, \widetilde{P}_{0 j}, \widetilde{P}_{j}$ :

$$
\left(\bar{P}_{j}\right)\left\{\begin{array}{l}
\bar{v}_{j}^{\prime \prime}\left(x^{e_{j}}\right)=f_{j}\left(x^{e_{j}}\right), x^{e_{j}} \in\left(\delta, l_{j}-\delta\right) \\
\bar{v}_{j}(\delta)=0 \\
\bar{v}_{j}\left(l_{j}-\delta\right)=0
\end{array}\right.
$$

$\left(\widetilde{P}_{j 0}\right)\left\{\begin{array}{l}\widetilde{v}_{j 0}^{\prime \prime}\left(x^{e_{j}}\right)=0, x^{e_{j}} \in\left(\delta, l_{j}-\delta\right) \\ \widetilde{v}_{j 0}(\delta)=1 \\ \widetilde{v}_{j 0}\left(l_{j}-\delta\right)=0\end{array} \quad\left(\widetilde{P}_{j j}\right)\left\{\begin{array}{l}\widetilde{v}_{j j}^{\prime \prime}\left(x^{e_{j}}\right)=0, x^{e_{j}} \in\left(\delta, l_{j}-\delta\right) \\ \widetilde{v}_{j j}(\delta)=0 \\ \widetilde{v}_{j j}\left(l_{j}-\delta\right)=1\end{array}\right.\right.$

$$
\left(\widetilde{P}_{0 j}\right)\left\{\begin{array} { l } 
{ \triangle \widetilde { u } _ { 0 j } ( x , y ) = 0 , ( x , y ) \in \Omega ( 0 ) } \\
{ \widetilde { u } _ { 0 j } | _ { \gamma _ { j } ^ { \prime } } = 1 , } \\
{ \widetilde { u } _ { 0 j } | _ { \gamma _ { k } ^ { \prime } } = 0 , \text { if } k \neq j , k = 1 , \ldots , n } \\
{ \frac { \partial u _ { 0 j } } { \partial n } = 0 , \text { on } \partial \Omega ( 0 ) \backslash ( \cup _ { j = 1 } ^ { n } \gamma _ { j } ^ { \prime } ) }
\end{array} \quad ( \widetilde { P } _ { j } ) \quad \left\{\begin{array}{l}
\triangle \widetilde{u}_{j}(x, y)=0,(x, y) \in \Omega(j) \\
\left.\widetilde{u}_{j}\right|_{\gamma_{j}^{\prime \prime}}=1, \\
\left.\widetilde{u}_{j}\right|_{\hat{\beta}_{j}^{\varepsilon}}=0, \\
\frac{\partial \widetilde{u}_{j}}{\partial n}=0, \text { on } \partial \Omega(j) \backslash\left(\gamma_{j}^{\prime \prime} \cup \hat{\beta}_{j}^{\varepsilon}\right)
\end{array}\right.\right.
$$

The boundary of the domain $\Omega_{\varepsilon}$ is smooth. Assume also that the following hypothesis holds:

(H1) Domain $\Omega(0)$ and its symmetric reflection $\Omega^{(i)}(0)$ with respect to the segment $\gamma_{i}^{\prime}$ (for sufficiently large $\delta / \varepsilon$ ) have the closures such that

$$
\overline{\Omega(0)} \cap \overline{\Omega^{(i)}(0)}=\overline{\gamma_{i}^{\prime}}
$$

for all $i=1, \ldots, n$.

The same property takes place for any $\Omega(i)$ with respect to $\gamma_{i}^{\prime \prime}$, but this property is not assumed: it is always satisfied for sufficiently large $\delta / \varepsilon$. 
Then using the extensions of problem $(3.4)\left(\tilde{P}_{0 j}\right)$ to $\Omega^{(i)}(0)$, odd with respect to $\gamma_{i}^{\prime}$, when $i \neq j$, and odd for $\widetilde{u}_{0 j}-1$, when $i=j$, it is easy to see that $\widetilde{u}_{0 j}$ is $C^{2}$-smooth.

Indeed, consider the extended problem in $\Omega(0) \cup \Omega^{(i)}(0) \cup \gamma_{i}^{\prime}: \Delta \tilde{u}_{0 j, \text { ext }}=0$ in this domain, $\frac{\partial \tilde{u}_{0 j, e x t}}{\partial n}=0$ for all boundary except for $\gamma_{k}^{\prime}(k \neq i)$ and their symmetric images $\gamma_{k}^{\prime(i)}$.

For $i \neq j$ we set: $\left.\tilde{u}_{0 j, e x t}\right|_{\gamma_{k}^{\prime} \cup \gamma_{k}^{\prime(i)}}=0$ for all $k \neq j ;\left.\tilde{u}_{0, j, e x t}\right|_{\gamma_{j}^{\prime}}=1,\left.\tilde{u}_{0, j, e x t}\right|_{\gamma_{j}^{\prime(i)}}=-1$.

For $i=j$ we set: $\left.\tilde{u}_{0 j, e x t}\right|_{\gamma_{k}^{\prime}}=0$ for all $k \neq j ;\left.\tilde{u}_{0 j, e x t}\right|_{\gamma_{k}^{\prime}(i)}=2$.

The uniqueness of the solution and its oddness for $i \neq j$ (and oddness of $\tilde{u}_{0 j, e x t}-1$ for $i=j$ ) with respect to $\gamma_{i}^{\prime}$ gives: $\left.\tilde{u}_{0, j, e x t}\right|_{\gamma_{i}^{\prime}}=0$ if $i \neq j$ and $\left.\tilde{u}_{0 j, e x t}\right|_{\gamma_{i}^{\prime}}=1$ if $i=j$. So, it coincides with $\tilde{u}_{0 j}$ on $\Omega(0)$. On the other hand, $\tilde{u}_{0 j, e x t}$ is smooth on the branch $B_{i}^{\varepsilon} \cap \Omega(0)$ (see [1]), and so $\tilde{u}_{0, j}$ is also smooth there up to the boundary, and consequently, everywhere in $\Omega(0)$ (even in the corners!).

Finally, all functions $\bar{v}_{j}, \widetilde{v}_{j 0}, \widetilde{v}_{j j}, \widetilde{u}_{0 j}, \widetilde{u}_{j}, \mathrm{j}=1, \ldots, \mathrm{n}$, are smooth. Let us note that $\left(\widetilde{P}_{j 0}\right)$ and $\left(\widetilde{P}_{j j}\right)$ can be solved analytically. We have $\widetilde{v}_{j 0}\left(x^{e_{j}}\right)=\frac{x^{e_{j}}-\left(l_{j}-\delta\right)}{2 \delta-l_{j}}, \widetilde{v}_{j j}\left(x^{e_{j}}\right)=$ $\frac{x^{e_{j}}-\delta}{l_{j}-2 \delta}=1-\widetilde{v}_{j 0}\left(x^{e_{j}}\right), x^{e_{j}} \in\left(\delta, l_{j}-\delta\right)$. This last relation leads to consider $\beta_{j}=\alpha_{0 . j}-\alpha_{j}, j=1, \ldots, n$. So, (3.1) is equivalent to:

$$
\left\{\begin{array}{l}
v_{j}=\bar{v}_{j}+\beta_{j} \widetilde{v}_{j 0}+\alpha_{j}, j=1, \ldots, n \\
u_{0}=\sum_{j=1}^{n-1}\left(\left(\alpha_{j}+\beta_{j}\right)-\left(\alpha_{n}+\beta_{n}\right)\right) \widetilde{u}_{0 j}+\left(\alpha_{n}+\beta_{n}\right) \\
u_{j}=\alpha_{j} \widetilde{u}_{j}, j=1, \ldots, n
\end{array}\right.
$$

Mention that $\sum_{j=1}^{n} \widetilde{u}_{0 j}=1$, so that $\widetilde{u}_{0 n}=1-\sum_{j=1}^{n-1} \widetilde{u}_{0 j}$.

It is easy to see that $v_{j}, u_{j}, j=1, \ldots, n$ satisfy $(2.2)$ if $\alpha_{j}, \beta_{j}, j=1, \ldots, n$ satisfy the relations

$$
\left\{\begin{array}{l}
\frac{1}{\theta_{j} \varepsilon} \sum_{k=1}^{n-1}\left(\left(\alpha_{k}+\beta_{k}\right)-\left(\alpha_{n}+\beta_{n}\right)\right) \int_{\gamma_{j}^{\prime}} \frac{\partial \widetilde{u}_{0 k}}{\partial n} d \gamma-\frac{\beta_{j}}{2 \delta-l_{j}}=\bar{v}_{j}^{\prime}(\delta), \\
j=1, \ldots, n-1 \\
-\frac{1}{\theta_{n}} \sum_{j=1}^{n-1} \theta_{j} \frac{\beta_{j}}{2 \delta-l_{j}}-\frac{\beta_{n}}{2 \delta-l_{n}}=\frac{1}{\theta_{n}} \sum_{j=1}^{n-1} \theta_{j} \bar{v}_{j}^{\prime}(\delta)+\bar{v}_{n}^{\prime}(\delta) \\
\alpha_{j} \frac{1}{\theta_{j} \varepsilon} \int_{\gamma_{j}^{\prime \prime}} \frac{\partial \widetilde{u}_{j}}{\partial n} d \gamma+\frac{\beta_{j}}{2 \delta-l_{j}}=-\bar{v}_{j}^{\prime}\left(l_{j}-\delta\right), j=1, \ldots, n
\end{array}\right.
$$

obtained by writing the interface conditions between the $1 \mathrm{D}$ parts and the $2 \mathrm{D}$ parts of the decomposed problem (the two last equations in (2.2)), and using

$$
v_{n}^{\prime}(\delta)=\frac{1}{\theta_{n} \varepsilon} \int_{\gamma_{n}^{\prime}} \frac{\partial u_{0}}{\partial n} d \gamma=-\frac{1}{\theta_{n} \varepsilon} \sum_{j=1}^{n-1} \int_{\gamma_{j}^{\prime}} \frac{\partial u_{0}}{\partial n} d \gamma=-\frac{1}{\theta_{n}} \sum_{j=1}^{n-1} \theta_{j} v_{j}^{\prime}(\delta)
$$

since $\int_{\Omega(0)} \triangle u_{0}=0=\sum_{j=1}^{n} \int_{\gamma_{j}^{\prime}} \frac{\partial u_{0}}{\partial n} d \gamma$. 
Let us simplify the notations. Define first

$$
r_{j k}=\frac{1}{\theta_{j} \varepsilon} \int_{\gamma_{j}^{\prime}} \frac{\partial \widetilde{u}_{0 k}}{\partial n} d \gamma, j, k=1, \ldots, n \quad t_{j}=\frac{1}{\theta_{j} \varepsilon} \int_{\gamma_{j}^{\prime \prime}} \frac{\partial \widetilde{u}_{j}}{\partial n} d \gamma, j=1, \ldots, n
$$

We have $\int_{\partial \Omega(j)} \widetilde{u}_{j} \frac{\partial \widetilde{u}_{j}}{\partial n} d \gamma=\int_{\Omega(j)}\left\|\nabla \widetilde{u}_{j}\right\|^{2}>0$, where $\|\cdot\|$ is the euclidian norm in $\mathbb{R}^{2}$ $\left(\widetilde{u}_{j}\right.$ is smooth, vanishes on $\hat{\beta}_{j}^{\varepsilon}$ and is equal to 1 on $\gamma_{j}^{\prime \prime}$, therefore $\left.\int_{\Omega(j)}\left\|\nabla \widetilde{u}_{j}\right\|^{2} \neq 0\right)$. We deduce

$$
\int_{\gamma_{j}^{\prime \prime}} \frac{\partial \widetilde{u}_{j}}{\partial n} d \gamma=\theta_{j} \varepsilon t_{j}>0, j=1, \ldots, n
$$

So, we just proved $t_{j}>0, j=1, \ldots, n$. Similarly one can get the inequalities $r_{j j}>0$, $j=1, \ldots, n$.

Now, we divide the lines $\mathrm{j}=1, \ldots, \mathrm{n}-1$ of (3.6) by $r_{j . j}$, and the lines $\mathrm{j}=\mathrm{n}+1, \ldots, 2 \mathrm{n}$ by $t_{j}$. We obtain the following system $\left(P_{\alpha}\right)$ below:

$$
\text { (3.8) }\left\{\begin{array}{l}
\sum_{k=1}^{n-1}\left(\left(\alpha_{k}+\beta_{k}\right)-\left(\alpha_{n}+\beta_{n}\right)\right) \frac{r_{j k}}{r_{j j}}-\frac{\beta_{j}}{\left(2 \delta-l_{j}\right) r_{j j}}=\frac{\bar{v}_{j}^{\prime}(\delta)}{r_{j j}}, j=1, \ldots, n-1 \\
-\sum_{j=1}^{n} \frac{\theta_{j}}{\theta_{n}} \frac{\beta_{j}}{2 \delta-l_{j}}=\sum_{j=1}^{n} \frac{\theta_{j}}{\theta_{n}} \bar{v}_{j}^{\prime}(\delta) \\
\alpha_{j}+\frac{\beta_{j}}{\left(2 \delta-l_{j}\right) t_{j}}=-\frac{\bar{v}_{j}^{\prime}\left(l_{j}-\delta\right)}{t_{j}}, j=1, \ldots, n
\end{array}\right.
$$

3.2. Uniqueness of the solution to $\left(P_{\alpha}\right)$. Indeed, $\sum_{k=1}^{n} \widetilde{u}_{0 k}=1$, so that $\sum_{k=1}^{n} r_{j k}=0, j=1, \ldots, n$. Then the $\mathrm{n}-1$ first equations of $\left(P_{\alpha}\right)$ can be written

$$
\sum_{k=1}^{n}\left(\alpha_{k}+\beta_{k}\right) r_{j k}-\frac{\beta_{j}}{2 \delta-l_{j}}=\bar{v}_{j}^{\prime}(\delta), j=1, \ldots, n-1 .
$$

Now, we remove from the n-th equation the sum of $\frac{\theta_{j}}{\theta_{n}}$ times each of the previous ones, $\mathrm{j}=1, \ldots, \mathrm{n}-1$. Denote $\left(P^{\prime}{ }_{\alpha}\right)$ the new system, equivalent to $\left(P_{\alpha}\right)$. The $\mathrm{n}$-th equation of $\left(P_{\alpha}^{\prime}\right)$ reads:

$$
-\frac{\beta_{n}}{2 \delta-l_{n}}-\sum_{j=1}^{n-1} \sum_{k=1}^{n}\left(\alpha_{k}+\beta_{k}\right) \frac{\theta_{j}}{\theta_{n}} r_{j k}=\bar{v}_{n}^{\prime}(\delta)
$$

Mention that $\int_{\Omega(0)} \triangle u_{0}=0=\sum_{j=1}^{n} \int_{\gamma_{j}^{\prime}} \frac{\partial u_{0}}{\partial n} d \gamma=\sum_{j=1}^{n} \sum_{k=1}^{n}\left(\alpha_{k}+\beta_{k}\right) \theta_{j} \varepsilon r_{j k}$. So, we get that $\left(P^{\prime}{ }_{\alpha}\right)$ reads

$$
\left\{\begin{array}{l}
\sum_{k=1}^{n}\left(\alpha_{k}+\beta_{k}\right) \theta_{j} \varepsilon r_{j k}-\frac{\theta_{j} \varepsilon \beta_{j}}{2 \delta-l_{j}}=\theta_{j} \varepsilon \bar{v}_{j}^{\prime}(\delta), j=1, \ldots, n \\
\theta_{j} \varepsilon \alpha_{j} t_{j}+\frac{\theta_{j} \varepsilon \beta_{j}}{2 \delta-l_{j}}=-\theta_{j} \varepsilon \bar{v}_{j}^{\prime}\left(l_{j}-\delta\right), j=1, \ldots, n
\end{array}\right.
$$


Now, since $\int_{\Omega(0)} u_{0} \triangle u_{0}=0=-\int_{\Omega(0)}\left\|\nabla u_{0}\right\|^{2}+\int_{\partial \Omega(0)} u_{0} \frac{\partial u_{0}}{\partial n} d \gamma$, we have

$$
\sum_{j=1}^{n} \int_{\gamma_{j}^{\prime}} u_{0} \frac{\partial u_{0}}{\partial n} d \gamma=\sum_{j=1}^{n}\left(\alpha_{j}+\beta_{j}\right) \sum_{k=1}^{n}\left(\alpha_{k}+\beta_{k}\right) \theta_{j} \varepsilon r_{j k} \geq 0
$$

The system $\left(P_{\alpha}^{\prime}\right)$ is linear. Let us write this system in the form $A^{\prime} U=B^{\prime}$ with $U^{T^{\prime}}=\left(\beta_{1}, \ldots, \beta_{n}, \alpha_{1}, \ldots, \alpha_{n}\right) .\left(P_{\alpha}\right)$ has a unique solution if and only if $\left(P^{\prime}{ }_{\alpha}\right)$ has a unique solution if and only if $A^{\prime} U=0 \Rightarrow U=0$. Let us set $T=\left[\begin{array}{cc}I_{n} & I_{n} \\ 0 & I_{n}\end{array}\right]$, a block matrix, where $I_{n}$ is the identity $n \times n$-matrix. We have

$A^{\prime} U=0 \Rightarrow(T U)^{T} A^{\prime} U=0 \Rightarrow \sum_{j=1}^{n}\left(\alpha_{j}+\beta_{j}\right) \sum_{k=1}^{n}\left(\alpha_{k}+\beta_{k}\right) \theta_{j} \varepsilon r_{j k}$

$-\sum_{j=1}^{n}\left(\alpha_{j}+\beta_{j}\right) \frac{\theta_{j} \varepsilon \beta_{j}}{2 \delta-l_{j}}+\sum_{j=1}^{n} \theta_{j} \varepsilon \alpha_{j}^{2} t_{j}+\sum_{j=1}^{n} \frac{\theta_{j} \varepsilon \alpha_{j} \beta_{j}}{2 \delta-l_{j}}=0$

The estimates (3.10) and (3.7) prove that $(T U)^{T} A^{\prime} U$ is a sum of positive terms $\left(l_{j}-2 \delta>0\right.$ since $\delta \rightarrow 0$ when $\varepsilon \rightarrow 0$.) We deduce that each term of the sum is zero, so that $\alpha_{j}=\beta_{j}=0, j=1, \ldots, n$, that is to say $U=0$. This proves that $\left(P_{\alpha}\right)$ has a unique solution.

4. Asymptotic solution of the Laplace equation in a truncated one bundle tube structure. In this section, we obtain estimates of $\widetilde{u}_{0 j}, \widetilde{u}_{j}, \mathrm{j}=1, \ldots, \mathrm{n}$, thanks to an asymptotic expansion. We also give estimates for the first and second derivatives. We conclude with estimates for $\alpha_{j}, \beta_{j}, \mathrm{j}=1, \ldots, \mathrm{n}$.

4.1. Preliminary estimates. Consider solution of the problem $\left(\widetilde{P}_{0 j}\right),(3.4)$. The domain $\Omega(0)$ depends on two small parameters $\varepsilon \rightarrow 0, \delta \rightarrow 0$, where $\frac{\delta}{\varepsilon}=\hat{K}|\ln (\varepsilon)|$, $\hat{K}$ is a constant independent of $\varepsilon, \delta$. It will be specified later. Namely, we are going to construct an asymptotic approximation $u_{j}^{(J)}$ of the solution of problem $\left(\widetilde{P}_{0 j}\right)$ of the accuracy of order $O\left(\varepsilon^{J}\right)$, and constant $\hat{K}$ depends on this order $J$ (see (4.5)).

Let $d_{j} \varepsilon$ be the minimal distance from the base of the cylinders $B_{j}^{\varepsilon}$ to a parallel crosssection $\sigma_{j}^{\varepsilon}$ of $B_{j}^{\varepsilon}$ such that this cross-section does not contain points of other cylinders (i.e. $\sigma_{j}^{\varepsilon} \cap B_{i}^{\varepsilon}=\emptyset, \quad i \neq j$ ). Consider function $\theta \in C^{4}\left(\mathbb{R}^{+}\right)$such that $\theta(t)=0$ for $t \leq d, \theta(t)=1$ for $t \geq 2 d$, where $d=\max \left\{d_{j}, 1 \leq j \leq n\right\}$. $\theta$ is independent of small parameters; define $T_{i}(\xi)=\theta\left(\xi_{1}^{e_{i}}\right)$ if $\varepsilon \xi \in B_{i}^{\varepsilon} ; T_{i}(\xi)=0$ if $\varepsilon \xi \notin B_{i}^{\varepsilon} ; \xi_{1}^{e_{i}}=\frac{x^{e_{i}}}{\varepsilon}$; $(x, y)=\varepsilon \xi$.

An asymptotic approximation $u_{j}^{(J)}$ is saught in the form of a linear function of the local variable $x^{e_{i}}$ for every branch $B_{i} \cap \Omega(0)$ (i.e. $\left.u_{j}^{(J)}=a_{i} x^{e_{i}}\right)$ multiplied by $\theta\left(\left|\frac{x^{e_{i}}}{\varepsilon}\right|\right.$ ) and completed by a boundary layer corrector $U$ depending on the variable $\xi$ and such that $U(\xi) \rightarrow$ const as $\xi_{1}^{e_{j}} \rightarrow+\infty$.

The linearity of $u_{j}^{(J)}$ at the branches follows from the analysis [14] section 4.3.1, where the dimensional reduction of the Laplace equation in a strip leads to the second order ordinary differential equation

$v^{\prime \prime}\left(x^{e_{j}}\right)=0$.

Thus, $u_{j}^{(J)}$ is saught in the form :

$$
u_{j}^{(J)}=\sum_{k \neq j} A_{k}\left(\frac{x^{e_{k}}}{\varepsilon} T_{k}\left(\frac{x}{\varepsilon}, \frac{y}{\varepsilon}\right) \theta_{k}^{-1}-\frac{x^{e_{j}}}{\varepsilon} T_{j}\left(\frac{x}{\varepsilon}, \frac{y}{\varepsilon}\right) \theta_{j}^{-1}+U_{k j}\left(\frac{x}{\varepsilon}, \frac{y}{\varepsilon}\right)\right)+A_{j}
$$


where $A_{k}$ are some constants, $U_{k j}$ is a solution of the following problem set in an unbounded domain

$\Omega_{\infty}(0)=\varepsilon^{-1}\{\Omega(0)\} \cup \cup_{k=1}^{n} B_{k}^{\infty}$

$B_{k}^{\infty}=\left\{(x, y) \in \mathbb{R}^{2}, x^{e_{k}} \in(0,+\infty), y^{e_{k}} \in\left(-\frac{\theta_{k}}{2}, \frac{\theta_{k}}{2}\right)\right\}:$

$$
\left\{\begin{array}{l}
\triangle_{\xi} U_{k j}(\xi)=F_{k j}(\xi), \xi \in \Omega_{\infty}(0) \\
\frac{\partial U_{k j}(\xi)}{\partial n_{\xi}}=0, \xi \in \partial \Omega_{\infty}(0) \\
U_{k j} \rightarrow 0, \xi_{1}^{e_{j}} \rightarrow+\infty \\
\lim _{\xi_{1} e_{l} \rightarrow+\infty} U_{k j}=\operatorname{const}(l), \xi \in B_{l}^{\infty}, l \neq j
\end{array}\right.
$$

where

$F_{k j}(\xi)=-\left(\xi_{1}^{e_{k}} T_{k}(\xi)\right)^{\prime \prime} \theta_{k}^{-1}+\left(\xi_{1}^{e_{j}} T_{j}(\xi)\right)^{\prime \prime} \theta_{j}^{-1}$ and $T_{k}(\xi)$ is extended as follows :

$T_{k}(\xi)=\left\{\begin{array}{l}\theta\left(\xi_{1}^{e_{k}}\right), \xi \in B_{k}^{\infty}, \\ 0, \xi \notin B_{k}^{\infty} .\end{array}\right.$

Let us remark that the right hand side of (4.1) doesn't contain $J$ explicitely. As we will see below, $J$ appears in the forthcoming estimates of the exponential decay of $U_{k j}$ and its derivatives, and so finally $J$ will define $\hat{K}$.

Mention that $\int_{\Omega(0)} F_{k j}(\xi) d \xi=-\theta_{k}^{-1} \theta_{k}+\theta_{j}^{-1} \theta_{j}=0$.

It is known (see [9]) that there exist a unique set of constants $a_{l k j}, k \neq j, l \neq j, l, k \in$ $\{1, \ldots, n\}$ and a function $U_{k j}$ satisfying the above problem and such that,

$$
\left|U_{k j}-a_{l k j}\right| \leq c_{1 j} e^{-c_{2 j} \xi_{1}^{e_{l}}}
$$

and

$$
\left|\nabla_{\xi} U_{k j}\right|,\left|\nabla_{\xi}^{2} U_{k j}\right| \leq c_{1 j} e^{-c_{2 j} \xi_{1}^{e_{l}}}
$$

at each branch $B_{l}^{\infty}, l \in\{1, \ldots, n\}, c_{1 j}, c_{2 j}>0$.

Here $a_{j k j}=0$.

Determine $\hat{K}$ from the inequality

$$
\hat{K} \geq \frac{J+1}{\max \left\{c_{2 j}, 1 \leq j \leq n\right\}} .
$$

This inequality yields:

$$
\text { if } \delta=\hat{K} \varepsilon|\ln (\varepsilon)| \text { then } e^{-c_{2 j} \frac{\delta}{\varepsilon}} \leq \varepsilon^{J+1}
$$

The constants $A_{k}, k \neq j$, are chosen in such a way that the boundary conditions at $\gamma_{i}^{\prime}$ for $u_{j}^{(J)}$ are satisfied with the accuracy $O\left(\varepsilon^{J}\right)$ :

$$
\left\{\begin{array}{l}
A_{j}+A_{i} \frac{\delta}{\varepsilon} \theta_{i}^{-1}+\sum_{k \neq j} A_{k} a_{i k j}=0, \text { if } i \neq j, i \in\{1, \ldots, n\}, \\
A_{j}-\sum_{k \neq j} A_{k} \frac{\delta}{\varepsilon} \theta_{j}^{-1}=1, \text { if } i=j .
\end{array}\right.
$$


Change the unknown $A_{j}$ to $A_{j} \frac{\delta}{\varepsilon} \theta_{j}$. Then the matrix of this linear system has a form $A^{(n)} \frac{\delta}{\varepsilon}+B^{(n)}$,

where $A^{(n)}, B^{(n)}$ are independent of $\varepsilon, \delta ; A^{(n)}$ has all elements equal to zero except for the $j-t h$ line, $j-t h$ column, and the diagonal. The diagonal is : $\left(\theta_{1}^{-1}, \ldots, \theta_{n}^{-1}\right)$, the $j-t h$ line has all elements (except for number $j$ )) equal to $-\theta_{j}^{-1}$, and the $j-t h$ column has all elements equal to $\theta_{j}^{-1}$. And $B_{i k}^{(n)}=\left(1-\delta_{i j}\right) a_{i k j}$.

Mention that $A^{(n)}$ is inversible because $\operatorname{det} A^{(n)}=\theta_{j}^{-1} \sum_{i=1}^{n} \Pi_{k \neq i} \theta_{k}^{-1}$.

Indeed, for any $n \geq 3$, let us permute the $j-t h$ line with the first, and the $j-t h$ column with the first one. Denote $\bar{\theta}_{i}=\theta_{i}$ if $i \neq 1, j ; \bar{\theta}_{1}=\theta_{j}$ and $\bar{\theta}_{j}=\theta_{1}$, and apply the induction.

Then

det $A^{(n)}$

$=\bar{\theta}_{1}^{-1} \operatorname{det}\left(\begin{array}{ccccc}1 & -\bar{\theta}_{1}^{-1} & -\bar{\theta}_{1}^{-1} & \ldots & -\bar{\theta}_{1}^{-1} \\ 1 & \bar{\theta}_{2}^{-1} & 0 & \ldots & 0 \\ 1 & 0 & \bar{\theta}_{3}^{-1} & \ldots & 0 \\ \cdot & \cdot & \cdot & \cdot & \cdot \\ 1 & 0 & 0 & \ldots & \bar{\theta}_{n}^{-1}\end{array}\right)$

$=\bar{\theta}_{1}^{-1}\left((-1)^{2 n} \bar{\theta}_{n}^{-1} \operatorname{det} A^{(n-1)} \bar{\theta}_{1}+(-1)^{n+1} \operatorname{det}\left(\begin{array}{ccccc}-\bar{\theta}_{1}^{-1} & -\bar{\theta}_{1}^{-1} & \ldots & -\bar{\theta}_{1}^{-1} & -\bar{\theta}_{1}^{-1} \\ \bar{\theta}_{2}^{-1} & 0 & \ldots & 0 & 0 \\ 0 & \bar{\theta}_{3}^{-1} & \ldots & 0 & 0 \\ \cdot & \cdot & \cdot & \cdot & \cdot \\ 0 & 0 & \ldots & \bar{\theta}_{n-1}^{-1} & 0\end{array}\right)\right)$

$=\bar{\theta}_{1}^{-1}\left(\bar{\theta}_{1} \bar{\theta}_{n}^{-1} \operatorname{det} A^{(n-1)}+(-1)^{n+1}(-1)^{n}\left(-\bar{\theta}_{1}^{-1}\right) \bar{\theta}_{2}^{-1} \ldots \bar{\theta}_{n-1}^{-1}\right)$

$=\bar{\theta}_{n}^{-1} \operatorname{det} A^{(n-1)}+\bar{\theta}_{1}^{-1} \Pi_{k=1}^{n-1} \bar{\theta}_{k}^{-1}$

$=\bar{\theta}_{1}^{-1}\left(\bar{\theta}_{n}^{-1} \sum_{i=1}^{n-1} \Pi_{k=1, k \neq i}^{n-1} \bar{\theta}_{k}^{-1}+\Pi_{k=1}^{n-1} \bar{\theta}_{k}^{-1}\right)$

$=\bar{\theta}_{1}^{-1} \sum_{i=1}^{n} \Pi_{k=1, k \neq i}^{n} \bar{\theta}_{k}^{-1}$

To initialize the induction let us check that

$\operatorname{det}\left(\begin{array}{cc}\bar{\theta}_{1}^{-1} & -\bar{\theta}_{1}^{-1} \\ \bar{\theta}_{1}^{-1} & \bar{\theta}_{2}^{-1}\end{array}\right)=\bar{\theta}_{1}^{-1}\left(\bar{\theta}_{1}^{-1}+\bar{\theta}_{2}^{-1}\right)$.

So, $\operatorname{det} A^{(n)}>0$, and so, for $\frac{\delta}{\varepsilon}$ large enough, $\operatorname{det}\left(A^{(n)} \frac{\delta}{\varepsilon}+B^{(n)}\right)>0$. Solving system (4.7), we get the vector $\vec{A}=\left(A_{1}, \ldots, A_{n}\right)^{T}$, such that $\left(A^{(n)} \frac{\delta}{\varepsilon}+B^{(n)}\right) \vec{A}=\vec{e}_{j}$, where $\vec{e}_{j}$ is the n-dimensional vector with the components equal to zero except for the component number $j$, that is, equal to one.

Let us show that $\|\vec{A}\|_{2}=O\left(\left(\frac{\delta}{\varepsilon}\right)^{-1}\right)\left(\|.\|_{2}\right.$ is the euclidian norm). Indeed,

$\frac{\delta}{\varepsilon} A^{(n)} \vec{A}=\vec{e}_{j}-B^{(n)} \vec{A}$,

$\overrightarrow{\vec{A}}=\frac{\varepsilon}{\delta}\left(A^{(n)}\right)^{-1} \vec{e}_{j}-\frac{\varepsilon}{\delta}\left(A^{(n)}\right)^{-1} B^{(n)} \vec{A}$,

$\|\vec{A}\|_{2} \leq \frac{\varepsilon}{\delta}\left\|\left(A^{(n)}\right)^{-1}\right\|_{2}\left\|\vec{e}_{j}\right\|_{2}+\frac{\varepsilon}{\delta}\left\|\left(A^{(n)}\right)^{-1}\right\|_{2}\left\|B^{(n)}\right\|_{2}\|\vec{A}\|_{2}$

and 
$\|\vec{A}\|_{2} \leq \frac{\varepsilon}{\delta} \frac{\left\|\left(A^{(n)}\right)^{-1}\right\|_{2}}{1-\frac{\varepsilon}{\delta}\left\|\left(A^{(n)}\right)^{-1}\right\|_{2}\left\|B^{(n)}\right\|_{2}}$

So, for $\frac{\delta}{\varepsilon}$ large enough, $\|\vec{A}\|_{2}=O\left(\frac{\varepsilon}{\delta}\right)$. Mention that $\frac{\delta}{\varepsilon}=\hat{K}|\ln (\varepsilon)| \rightarrow+\infty$.

Mention that the constructed asymptotic approximation (4.1) satisfies equation and conditions $\left(\widetilde{P}_{0 j}\right)$ exactly, except for the boundary conditions at $\gamma_{i}^{\prime}$ : due to the properties (4.3), (4.4), and the choice of $\hat{K}$ satisfying (4.5), (4.6), they are satisfied up to the terms of order $O\left(\varepsilon^{J+1}\right) \max \left\{A_{k}, 1 \leq k \leq n\right\}=O\left(\varepsilon^{J+1} \frac{\varepsilon}{\delta}\right)$ in the $H^{1 / 2}\left(\gamma_{i}^{\prime}\right)$ norm. Extending these traces $\left.u_{j}^{(J)}\right|_{\gamma_{i}^{\prime}}$ from $\gamma_{i}^{\prime}$ to each $B_{i}^{\varepsilon} \cap \Omega(0)$ as

$$
\left\{\begin{array}{l}
\sum_{k \neq j} A_{k}\left(U_{k j}\left(\frac{x}{\varepsilon}, \frac{y}{\varepsilon}\right)-a_{i k j}\right)\left(1-\theta\left(\frac{x^{e_{i}}-\delta}{\varepsilon}\right)\right), \text { if }\left|x^{e_{i}}-\delta\right|<\varepsilon \\
0, \text { if }\left|x^{e_{i}}-\delta\right| \geq \varepsilon,\left(a_{j k j}=0\right)
\end{array}\right.
$$

we check directly that the difference $W$ between $u_{j}^{(J)}$ and this extension satisfies $\left(\widetilde{P}_{0 j}\right)$ with the non-vanishing right hand side in the Laplace equation of order $O\left(\varepsilon^{J}\right)$ in $H^{-1}(\Omega(0))$. Taking into consideration the Poincaré-Friedrichs inequality in $\Omega(0)$ (for functions of $H^{1}\left(\Omega(0)\right.$ ) vanishing at $\cup_{i=1}^{n} \gamma_{i}^{\prime}$ ) with the constant independent of $\varepsilon, \delta$ (see [14], Ch4, Appendix), we get the estimate (following the Lax-Milgram lemma): $\left\|\widetilde{u}_{0 j}-W\right\|_{H^{1}(\Omega(0))}=O\left(\varepsilon^{J}\right)$, and so, $\left\|u_{j}^{(J)}-\widetilde{u}_{0 j}\right\|_{H^{1}(\Omega(0))}=O\left(\varepsilon^{J}\right)$.

Remark 4.1. The same analysis for problems $\left(\widetilde{P}_{j}\right),(3.4)$, gives an asymptotic approximation in the form

$\widetilde{u}_{j}^{(J)}=\widetilde{A}_{j}\left(\frac{\hat{x}^{e_{j}}}{\varepsilon} \theta\left(\frac{\hat{x}^{e_{j}}}{\varepsilon}\right)+U_{j}\left(\frac{x-O_{j}}{\varepsilon}\right)\right)$

where $\widetilde{A}_{j}$ is a constant, $U_{j}$ is a solution of the following problem set in an unbounded domain $\Omega_{\infty}(j)=\varepsilon^{-1}\left\{\Omega(j)-O_{j}\right\} \cup \widetilde{B}_{j}^{\infty}$, where

$\widetilde{B}_{j}^{\infty}=\left\{(x, y) \in \mathbb{R}^{2}, \hat{x}^{e_{j}} \in(0,+\infty), \hat{y}^{e_{j}} \in\left(-\frac{\theta_{j}}{2}, \frac{\theta_{j}}{2}\right)\right\}:$

$$
\left\{\begin{array}{l}
\triangle_{\xi} U_{j}(\xi)=F_{j}(\xi), \xi \in \Omega_{\infty}(j), \\
\frac{\partial U_{j}(\xi)}{\partial n_{\xi}}=0, \xi \in \partial \Omega_{\infty}(j) \backslash \widetilde{\beta}_{j} \\
\left.U_{j}\right|_{\widetilde{\beta}_{j}}=0, \\
\lim _{\hat{\xi}_{1}^{e} \rightarrow+\infty} U_{j}=\text { const. }
\end{array}\right.
$$

Here $\widetilde{\beta}_{j}=\varepsilon^{-1}\left\{\hat{\beta}_{j}^{\varepsilon}-O_{j}\right\}, F_{j}=-\left(\hat{\xi}_{1}^{e_{j}} \theta\left(\hat{\xi}_{1}^{e_{j}}\right)\right)^{\prime \prime}$.

$\widetilde{A}_{j}$ is chosen from the conditions of vanishing of $\widetilde{u}_{j}^{(J)}$ on $\hat{\beta}_{j}^{\varepsilon}$ and $\widetilde{u}_{j}^{(J)}=1$ on $\gamma_{j}^{\prime \prime}$ :

$\widetilde{A}_{j}\left(\frac{\delta}{\varepsilon}+\lim _{\hat{\xi}_{1}^{e_{j}} \rightarrow+\infty} U_{j}\right)=1$, i.e. $\widetilde{A}_{j}=\frac{1}{\frac{\delta}{\varepsilon}+\lim _{\hat{\xi}_{1}^{e_{j} \rightarrow+\infty}} U_{j}}=O\left(\frac{\varepsilon}{\delta}\right)$.

As for $\widetilde{u}_{0 j}$ we have : if $\delta=K \varepsilon|\ln (\varepsilon)|$ then $\left\|\widetilde{u}_{j}-\widetilde{u}_{j}^{(J)}\right\|_{H^{1}(\Omega(j))}=O\left(\varepsilon^{J+1}\right)$.

Here $K$ is chosen from the condition $K \geq \frac{J+1}{C_{2 j}}$, where $C_{2 j}$ is such that

$\left|U_{j}-\lim _{\hat{\xi}_{1}^{e_{j}} \rightarrow+\infty} U_{j}\right| \leq C_{1 j} e^{-C_{2 j} \hat{\xi}_{1}^{e_{j}}}$ and $\left|\nabla U_{j}\right|,\left|\nabla^{2} U_{j}\right| \leq C_{1 j} e^{-C_{2 j} \hat{\xi}_{1}^{e_{j}}}$. 
4.2. Estimates for the second derivatives. Here we will improve the estimates for $\left\|u_{j}^{(J)}-\tilde{u}_{0 j}\right\|_{H^{1}}$ and $\left\|\tilde{u}_{j}^{(J)}-\tilde{u}_{j}\right\|_{H^{1}}$ and get the estimates for $\left\|\nabla\left(u_{j}^{(J)}-\tilde{u}_{0 j}\right)\right\|_{L^{\infty}}$ and $\left\|\nabla^{2}\left(u_{j}^{(J)}-\tilde{u}_{0 j}\right)\right\|_{L^{\infty}}$ and finally $\left\|\nabla \tilde{u}_{0 j}\right\|_{L^{\infty}}$ and $\left\|\nabla^{2} \tilde{u}_{0 j}\right\|_{L^{\infty}}$ and the same estimates for $\tilde{\tilde{u}}_{j}$. As it was just mentioned above, the hypothesis (H1) is assumed and it implies the smoothness of the exact solution. On the other hand, by construction, the asymptotic solution $u_{j}^{(J)}$ as well as function $W$ are also the $C^{2}$-smooth functions and $W$ can be extended to $\Omega^{(i)}(0)$ in the same way as it was done for the exact solution $\tilde{u}_{0 j}$. So, the difference $W-\tilde{u}_{0 j}$ in any extended domain $\Omega(0) \cup \Omega^{(i)}(0) \cup \gamma_{i}^{\prime}$ : satsfies the Laplace equation with a small right hand side of order $O\left(\varepsilon^{J-1}\right)$. Then, applying the Agmon-Douglis-Nirenberg estimates [1] to this difference $\tilde{u}_{0 j}-W$, we get the following estimates:

$$
\begin{gathered}
\left\|W-\tilde{u}_{0 j}\right\|_{H^{4}(\Omega(0))}=O\left(\varepsilon^{J-3}\right), \\
\left\|u_{j}^{(J)}-\tilde{u}_{0 j}\right\|_{H^{4}(\Omega(0))}=O\left(\varepsilon^{J-3}\right),
\end{gathered}
$$

and so,

$$
\left\|\nabla\left(u_{j}^{(J)}-\tilde{u}_{0 j}\right)\right\|_{C(\overline{\Omega(0)})}=O\left(\varepsilon^{J-3}\right)
$$

and

$$
\left\|\nabla^{2}\left(u_{j}^{(J)}-\tilde{u}_{0 j}\right)\right\|_{C(\overline{\Omega(0)})}=O\left(\varepsilon^{J-3}\right) .
$$

And so, taking $J \geq 4$, we see that

$$
\begin{gathered}
\left\|\nabla \tilde{u}_{0 j}\right\|_{C(\overline{\Omega(0)})}=\left\|\nabla u_{j}^{(J)}\right\|_{C(\overline{\Omega(0)})}+O(\varepsilon)=O\left(\frac{\varepsilon}{\delta} \varepsilon^{-1}\right)=O\left(\frac{1}{\delta}\right), \\
\left\|\nabla^{2} \tilde{u}_{0 j}\right\|_{C(\overline{\Omega(0)})}=\left\|\nabla^{2} u_{j}^{(J)}\right\|_{C(\overline{\Omega(0)})}+O(\varepsilon)=O\left(\frac{\varepsilon}{\delta} \varepsilon^{-2}\right)=O\left(\frac{1}{\varepsilon \delta}\right) .
\end{gathered}
$$

The same calculations for $\tilde{u}_{j}$ give

$$
\left\|\nabla \tilde{u}_{j}\right\|_{C(\overline{\Omega(j)})}=O\left(\frac{1}{\delta}\right),\left\|\nabla^{2} \tilde{u}_{j}\right\|_{C(\overline{\Omega(j)})}=O\left(\frac{1}{\varepsilon \delta}\right), j=1, \ldots, n .
$$

4.3. Estimates for $\alpha_{j}, \beta_{j}, \quad j=1, \ldots, n$. Let get the estimates for $\alpha_{j}, \beta_{j}$. Rewrite $\left(P_{\alpha}\right)$ in the form of a system $A U=B$ with block matrices $A, B$ defined by $A=\left[\begin{array}{cc}M & C \\ D & I_{n}\end{array}\right]$, and $B=\left[\begin{array}{c}B 1 \\ B 2\end{array}\right]$. The matrices $M, C, D$ are of dimension $n \times n$ and $B 1, B 2$ are the $n \times 1-$ columns. 
Denote $\delta_{j k}=1$ if $k=j$ and 0 else, $j=1, \ldots, n$, and define $M=\left(M_{j k}\right)_{1 \leq j, k \leq n}$ with

$$
\begin{aligned}
M_{j k} & =\frac{r_{j k}}{r_{j j}}-\frac{\delta_{j k}}{\left(2 \delta-l_{j}\right) r_{j j}} \text { if } j, k=1, \ldots, n-1 \\
& =-\sum_{i=1}^{n-1} \frac{r_{j i}}{r_{j j}} \text { if } j=1, \ldots, n-1, k=n \\
& =-\frac{\theta_{k}}{\theta_{n}} \frac{1}{\left(2 \delta-l_{k}\right)} \text { if } j=n, k=1, \ldots, n
\end{aligned}
$$

$C=\left(C_{j k}\right)_{1 \leq j, k \leq n}$ with

$$
\begin{aligned}
C_{j k} & =\frac{r_{j k}}{r_{j j}} \text { if } j, k=1, \ldots, n-1 \\
& =-\sum_{i=1}^{n-1} \frac{r_{j i}}{r_{j j}} \text { if } j=1, \ldots, n-1, k=n \\
& =0 \text { if } j=n, k=1, \ldots, n
\end{aligned}
$$

$D=\left(D_{j k}\right)_{1 \leq i, k \leq n}$ with

$$
D_{j k}=d_{j} \delta_{j k}, j, k=1, \ldots, n, \quad d_{j}=\frac{1}{\left(2 \delta-l_{j}\right) t_{j}}, j=1, \ldots, n
$$

$B 1=\left(B 1_{j}\right)_{1 \leq j \leq n}$ and $B 2=\left(B 2_{j}\right)_{1 \leq j \leq n}$ with

$$
B 1_{j}=\frac{\bar{v}_{j}^{\prime}(\delta)}{r_{j j}}, j=1, \ldots, n-1, \text { and } B 1_{n}=\sum_{i=1}^{n} \frac{\theta_{i}}{\theta_{n}} \bar{v}_{i}^{\prime}(\delta)
$$

We still denote $\alpha^{T}=\left(\alpha_{1}, \ldots, \alpha_{n}\right)$ and $\beta^{T}=\left(\beta_{1}, \ldots, \beta_{n}\right)$.

$A U=B \Leftrightarrow\left\{\begin{array}{l}M \beta+C \alpha=B 1 \\ D \beta+\alpha=B 2\end{array} \Leftrightarrow\left\{\begin{array}{l}(M-C D) \beta=B 1-C B 2 \\ \alpha=B 2-D \beta\end{array}\right.\right.$

Present $M-C D$ in the form $M-C D=A_{0}+\delta A_{1}$ where $A_{0}=\left(A_{0 j k}\right)_{1 \leq j, k \leq n}$ and $A_{1}=\left(A_{1 j k}\right)_{1 \leq j, k \leq n}$ defined by

$$
\begin{gathered}
A_{0 j k}=\frac{r_{j k}}{r_{j, j}} \text { if } j, k=1, \ldots, n-1 \\
=-\sum_{i=1}^{n-1} \frac{r_{j i}}{r_{j j}} \text { if } j=1, \ldots, n-1, k=n \\
=-\frac{\theta_{k}}{\theta_{n}} \frac{1}{\left(2 \delta-l_{k}\right)} \text { if } j=n, k=1, \ldots, n \\
\delta A_{1 j k}=-\frac{\delta_{j k}}{\left(2 \delta-l_{j}\right) r_{j . j}}-\frac{r_{j k}}{r_{j . j}} \frac{1}{\left(2 \delta-l_{k}\right) t_{k}} \text { if } j, k=1, \ldots, n-1 \\
=\left(\sum_{i=1}^{n-1} \frac{r_{j i}}{r_{j j}}\right) \frac{1}{\left(2 \delta-l_{n}\right) t_{n}} \text { if } j=1, \ldots, n-1, k=n \\
=0 \text { if } j=n, k=1, \ldots, n
\end{gathered}
$$


We deduce from the above asymptotic analysis that $r_{j k}=O\left(\frac{1}{\delta}\right), j, k=1, \ldots, n$; we prove that $\frac{1}{r_{j j}}=O(\delta), \frac{1}{t_{j}}=O(\delta), j=1, \ldots, n$. So $A_{0}=O(1), A_{1}=O(1), B 1=O(1)$ because of its $n^{\text {th }}$ component, and $B 2=O(\delta)$. We have $C=O(1), D=O(\delta)$.

$A_{0}$ is a regular matrix. Indeed, if we add the $n^{\text {th }}$ column of the determinant of $A_{0}$, to the sum of the previous ones, we obtain

$\operatorname{det}\left(A_{0}\right)=\frac{\sum_{i=1}^{n}-\frac{\theta_{j}}{\theta_{n}} \frac{1}{2 \delta-l_{j}}}{r_{11} r_{22} \ldots r_{n-1, n-1}} \operatorname{det}(R)$ with $R=\left(r_{j k}\right)_{1 \leq j, k \leq n-1}$.

$A_{0}$ is a regular matrix if and only if $R$ is regular too i.e., if and only if $R X=0 \Rightarrow$ $X=0$. Set $X^{T}=\left(x_{1}, \ldots, x_{n-1}\right)$, denote $v=\sum_{k=1}^{n-1} x_{k} \widetilde{u}_{0 k}$ and mention that $v$ satisfies the following problem

$$
\left\{\begin{array}{l}
\triangle v=0, \text { on } \Omega(0) \\
\left.v\right|_{\gamma_{j}^{\prime}}=x_{j}, j=1, \ldots, n-1,\left.v\right|_{\gamma_{n}^{\prime}}=0 \\
\frac{\partial v}{\partial n}=0, \text { on } \partial \Omega(0) \backslash\left(\cup_{j=1}^{n} \gamma_{j}^{\prime}\right) \\
\int_{\gamma_{j}^{\prime}} \frac{\partial v}{\partial n} d \gamma=\sum_{k=1}^{n-1} x_{k} \theta_{j} \varepsilon r_{j k}=0, j=1, \ldots, n-1
\end{array}\right.
$$

Now, we have the identity:

$\int_{\Omega(0)} v \triangle v=0=-\int_{\Omega(0)}\|\nabla v\|^{2}+\int_{\partial \Omega(0)} v \frac{\partial v}{\partial n} d \gamma$, so

$\int_{\Omega(0)}\|\nabla v\|^{2}=\sum_{j=1}^{n} \int_{\gamma_{j}^{\prime}} v \frac{\partial v}{\partial n} d \gamma=\sum_{j=1}^{n-1} x_{j} \int_{\gamma_{j}^{\prime}} \frac{\partial v}{\partial n} d \gamma=0$

So, $v$ is a constant function on $\Omega(0)$, and necessarily we have $X=0$.

This proves $A_{0}$ is a regular matrix. Since $\delta$ tends to 0 , we can assume $\left\|\delta A_{0}^{-1} A_{1}\right\|<1$ and obtain the estimate for $\beta$.

$\beta=\left(A_{0}+\delta A_{1}\right)^{-1}(B 1-C B 2)=\left(I_{n}+\delta A_{0}^{-1} A_{1}\right)^{-1} A_{0}^{-1}(B 1-C B 2)$

$=\left(\sum_{k=0}^{\infty}(-1)^{k} \delta^{k}\left(A_{0}^{-1} A_{1}\right)^{k}\right) A_{0}^{-1}(B 1-C B 2)$. We conclude that

$\|\beta\| \leq\left(\sum_{k=0}^{\infty} \delta^{k}\left\|A_{0}^{-1} A_{1}\right\|^{k}\right)\left\|A_{0}^{-1}(B 1-C B 2)\right\| \leq \frac{1}{1-\delta\left\|A_{0}^{-1} A_{1}\right\|}\left\|A_{0}^{-1}(B 1-C B 2)\right\|$ So $\beta=O(1)$ but $\alpha=B 2-D \beta=O(\delta)$.

5. Numerical scheme. In this section we solve $\left(\bar{P}_{j}\right), \mathrm{j}=1, \ldots, \mathrm{n}$, by a $1 \mathrm{D}$-finite volume method, solve $\left(\widetilde{P}_{0 j}\right)$ and $\left(\widetilde{P}_{j}\right), \mathrm{j}=1, \ldots, \mathrm{n}$, by a 2 D-finite volume method, and finally solve the algebraic system (3.8).

The finite volume method is often applied to obtain solution of some elliptic and parabolic problems [19]. Though the method was first apply to solve hyperbolic equations, corresponding to some conservation laws (see for instance [18]).

In [22], the author presents a general framework to discretize elliptic problems by applying two different finite volume schemes in two different subdomains. In [23], the authors study asymptotic model of fracture flow in a bidimensionnel porous medium : the fractures are reduced to sharp interfaces when the fracture aperture goes to zero, and the model finally consists in a coupling between a $2 \mathrm{D}$ elliptic problem and a $1 \mathrm{D}$ equation on the sharp interfaces. This approach differs from the MAPDD because it studies the problem obtained after some complete dimensional reduction, while the MAPDD "makes a zoom" at the boundary layer zones.

5.1. Numerical approximation of $\left(\bar{P}_{j}\right)$. In order to compute a numerical approximation $\bar{v}_{j} \mathcal{T}$ of $\bar{v}_{j}$, let us define a mesh of the interval $\left(\delta, l_{j}-\delta\right)$ on the axis $O x^{e_{j}}, j=1, \ldots, n$. For each value of $\mathrm{j}=1, \ldots, \mathrm{n}$, we choose $N_{j} \in \mathbb{N}^{*}$, and $N_{j}+1$ distinct 
and increasing values $x_{i+1 / 2}^{e_{j}}, i=0, \ldots, N_{j}$, such that $x_{1 / 2}^{e_{j}}=\delta, x_{N_{j}+1 / 2}^{e_{j}}=l_{j}-\delta$. Denote $I_{i}^{e_{j}}=\left(x_{i-1 / 2}^{e_{j}}, x_{i+1 / 2}^{e_{j}}\right)$, and $h_{i}^{e_{j}}=x_{i+1 / 2}^{e_{j}}-x_{i-1 / 2}^{e_{j}}, i=1, \ldots, N_{j}$.

Set $h^{e_{j}}=\max \left(h_{i}^{e_{j}}, i=1, \ldots, N_{j}\right)$ the step of the mesh.

Then we choose $N_{j}$ points $x_{i}^{e_{j}}, i=1, \ldots, N_{j}$, such that $x_{i}^{e_{j}} \in I_{i}^{e_{j}}$. Set $x_{0}^{e_{j}}=\delta, x_{N_{j}+1}^{e_{j}}=$ $l_{j}-\delta . h_{i+1 / 2}^{e_{j}}=x_{i+1}^{e_{j}}-x_{i}^{e_{j}}, i=0, \ldots, N_{j}$.

Consider the finite volume $1 \mathrm{D}$ scheme for problem $\left(\bar{P}_{j}\right), \mathrm{j}=1, \ldots, \mathrm{n}$. This scheme is obtained by the integrating $\bar{v}_{j}^{\prime \prime}=f_{j}$ on each cell $I_{i}^{e_{j}}, i=1, \ldots, N_{j}$. The numerical flux $F_{i+1 / 2}$ is an approximation of $\bar{v}_{j}^{\prime}\left(x_{i+1 / 2}^{e_{j}}\right)$ of finite difference type; $\bar{v}_{j, i}$ is an approximation of $\bar{v}_{j}\left(x_{i}^{e_{j}}\right), i=0, \ldots, N_{j}+1$. This yields

$$
(S j D 1)\left\{\begin{array}{l}
F_{i+1 / 2}-F_{i-1 / 2}=h_{i}^{e_{j}} f_{i}^{e_{j}}, i=1, \ldots, N_{j} \\
F_{i+1 / 2}=\frac{\bar{v}_{j, i+1}-\bar{v}_{j, i}}{h_{i+1 / 2}^{e_{j}}}, i=0, \ldots, N_{j} ; \bar{v}_{j, 0}=\bar{v}_{j, N_{j}+1}=0, \\
f_{i}^{e_{j}}=\frac{1}{h_{i}^{e_{j}}} \int_{x_{i-1 / 2}^{e_{j}}}^{x_{i+1 / 2}^{e_{j}}} f_{j}(x) d x, i=1, \ldots, N_{j}
\end{array}\right.
$$

If $W$ is a piecewise constant function, that is $W\left(x^{e_{j}}\right)=W_{i}, x^{e_{j}} \in I_{i}^{e_{j}}, i=$ $1, \ldots, N_{j}$, then we will denote by $\|W\|_{j, \mathcal{T}}=\left(\sum_{i=1}^{N_{j}-1} \frac{\left(W_{i+1}-W_{i}\right)^{2}}{h_{i+1 / 2}^{e_{j}}}+\frac{W_{1}^{2}}{h_{1 / 2}^{e_{j}}}+\frac{W_{N_{j}}^{2}}{h_{N_{j}+1 / 2}^{e_{j}}}\right)^{1 / 2}$, that can be seen as a discrete norm in $H_{0}^{1}\left(\delta, l_{j}-\delta\right)$.

Denote by $\|\cdot\|_{L^{2}\left(\delta, l_{j}-\delta\right)}$ the discrete norm in $L^{2}\left(\delta, l_{j}-\delta\right)$, i.e., $\|W\|_{L^{2}\left(\delta, l_{j}-\delta\right)}=\left(\sum_{i=1}^{N_{1}} h_{i}^{e_{j}} W_{i}^{2}\right)^{1 / 2}$. Define $\bar{v}_{j \mathcal{T}}\left(x^{e_{j}}\right)=\bar{v}_{j, i}, x^{e_{j}} \in I_{i}^{e_{j}}, i=1, \ldots, N_{j}$, and the error $\bar{e}_{j \mathcal{T}}$ by $\bar{e}_{j \mathcal{T}}\left(x^{e_{j}}\right)=$ $\bar{v}_{j}\left(x_{i}^{e_{j}}\right)-\bar{v}_{j, i}, x^{e_{j}} \in I_{i}^{e_{j}}, i=1, \ldots, N_{j}$.

In [19] the estimate $\left\|\bar{e}_{j \mathcal{T}}\right\|_{j, \mathcal{T}} \leq c_{j} h^{e_{j}}$ is proved, and it implies

$$
\left\|\bar{v}_{j}-\bar{v}_{j} \mathcal{T}\right\|_{L^{2}\left(\delta, l_{j}-\delta\right)} \leq C_{j} h^{e_{j}}, j=1, \ldots, n
$$

where $C_{j}$ is a constant independent of $\delta$ and $\varepsilon$.

5.2. Numerical approximation of $\left(\widetilde{P}_{0 j}\right)$ and $\left(\widetilde{P}_{j}\right)$. Let us construct an admissible mesh $\mathcal{T}_{j}$ over $\Omega(j), \mathrm{j}=0, \ldots, \mathrm{n}$. Remind that such mesh consists of subsets $K$ (called control volumes) such that either $K$ is a polygonal domain of $\mathbb{R}^{2}$, or it is an intersection of a polygonal domain and $\Omega(j)$ (such control volumes are situated near the curved part of the boundary).

Let $\mathcal{E}$ be the family of edges $\sigma$ of the control volumes, and $P$ be a family of points $x_{K}$ chosen in each control volume $K$. 
$\mathcal{T}_{j}$ satisfies the following properties

1) the closure of the union of all the control volumes is $\overline{\Omega(j)}$.

2 ) for any $K \in \mathcal{T}_{j}$, there exists a subset $\mathcal{E}_{K}$ of $\mathcal{E}$ such that

$\partial K=\bigcup_{\sigma \in \mathcal{E}_{K}} \bar{\sigma}$, and $\bigcup_{K \in \mathcal{T}_{j}} \mathcal{E}_{K}=\mathcal{E}$.

3) $\forall(K, L) \in \mathcal{T}_{j}^{2}, K \neq L$, one of three following assertions holds:

either $\bar{K} \cap \bar{L}$ is the empty set, or $\bar{K} \cap \bar{L}$ is a common vertex of $\mathrm{K}$ and $\mathrm{L}$,

(5.3) or $\bar{K} \cap \bar{L}$ is a common edge of $\mathrm{K}$ and $\mathrm{L}$ denoted by $\sigma_{K / L}$.

4) Let $P=\left(x_{K}\right)_{K \in \mathcal{T}_{j}}$. We assume $x_{K} \in \bar{K}$, for any $K \in \mathcal{T}_{j}$.

$\forall(K, L) \in \mathcal{T}_{j}^{2}$, if $K \neq L$, then $x_{K} \neq x_{L}$ and the straight line going

through $x_{K}$ and $x_{L}$ is orthogonal to $\sigma_{K / L}$.

5) $\forall \sigma \in \mathcal{E}$, if $\sigma \subset \partial \Omega(j), \sigma \in \mathcal{E}_{K}$ and $x_{K} \notin \sigma$, then the orthogonal projection of $x_{K}$ on the straight line containing the edge $\sigma$, belongs to this edge $\sigma$

Denote by $\mathcal{E}_{\text {int }}=\{\sigma \in \mathcal{E}, \sigma \not \subset \partial \Omega(j)\}$. Denote by $d_{\sigma}$ the distance between $x_{K}$ and $x_{L}$ if $\sigma=\sigma_{K / L}$, for any $(K, L) \in \mathcal{T}_{j}^{2}(K \neq L)$, and $d_{\sigma}$ stands for the distance between $x_{K}$ and $\sigma$, if $\sigma \in \mathcal{E}_{K}$ and if $\sigma \subset \partial \Omega(j)$, for any $K \in \mathcal{T}_{j}$.

Let $m(K)$ be the area of $K$, for any $K \in \mathcal{T}_{j}$.

Let $m(\sigma)$ be the length of $\sigma$, for any $\sigma \in \mathcal{E}$.

Let $h_{j}$ be the size of the mesh $\mathcal{T}_{j}$. Moreover, we assume

$$
\exists \zeta>0, \forall j \in\{1, \ldots, n\}, \forall \sigma \subset \gamma_{j}^{\prime}, d_{\sigma} \geq \zeta h_{0} \text { and } \forall \sigma \subset \gamma_{j}^{\prime \prime}, d_{\sigma} \geq \zeta h_{j}
$$

where $\zeta$ is independant of $h_{j}, j \in\{0, \ldots, n\}$.

Consider first $\widetilde{P}_{0 j}, j=1, \ldots, n$ and a numerical approximation $\widetilde{u}_{0 j \mathcal{T}}$ of $\widetilde{u}_{0 j}$. As in the $1 \mathrm{D}$ case, the finite volume scheme is obtained by integrating the first equation $\triangle \widetilde{u}_{0 j}=0$ over each control volume $K \in \mathcal{T}_{0}$ and by approximating the fluxes by corresponding differential quotients. Let $\widetilde{u}_{0 j K}$ be an approximation of $\widetilde{u}_{0 j}\left(x_{K}\right), K \in$ $\mathcal{T}_{0}$. Then for instance, the flux $F_{K, \sigma}$ through the edge $\sigma_{K / L}$, from the cell $K$ to the cell $L$, will be approximated as follows: $\int_{\sigma_{K / L}} \frac{\partial \widetilde{u}_{0 j}}{\partial n} d \gamma \approx \frac{m(\sigma)}{d_{\sigma}}\left(\widetilde{u}_{0 j L}-\widetilde{u}_{0 j K}\right)$. Here $m(\sigma)$ is the length of $\sigma$.

A $2 \mathrm{D}$ - finite volume scheme with the unknown function $\widetilde{u}_{0 j \mathcal{T}}$ can be defined by the following set of equations:

$$
(S 0 j D 2)\left\{\begin{aligned}
& \sum_{\sigma \in \mathcal{E}_{K}} F_{K, \sigma}=0, \forall K \in \mathcal{T}_{0} \\
& F_{K, \sigma}= \frac{m(\sigma)}{d_{\sigma}}\left(\widetilde{u}_{0 j L}-\widetilde{u}_{0 j K}\right), \forall \sigma \in \mathcal{E}_{i n t}, \text { if } \sigma=\sigma_{K / L} \\
& F_{K, \sigma}=\frac{m(\sigma)}{d_{\sigma}}\left(1-\widetilde{u}_{0 j K}\right), \forall \sigma \subset \gamma_{j}^{\prime}, \sigma \in \mathcal{E}_{K} \\
&=\frac{m(\sigma)}{d_{\sigma}}\left(-\widetilde{u}_{0 j K}\right), \forall \sigma \subset \gamma_{k}^{\prime}, k \neq j, \sigma \in \mathcal{E}_{K} \\
&=0, \quad \forall \sigma \subset \partial \Omega(0) \backslash\left(\cup_{j=1}^{n} \gamma_{j}^{\prime}\right), \sigma \in \mathcal{E}_{K}
\end{aligned}\right.
$$

The flux $F_{K, \sigma}$ is an auxiliary unknown, and after its elimination, we obtain a linear system with as many unknowns as equations $\left(\operatorname{card}\left(\mathcal{T}_{0}\right)\right)$.

We define $\widetilde{u}_{0 j \mathcal{T}}(x, y)=\widetilde{u}_{0 j K},(x, y) \in K, K \in \mathcal{T}_{0}, j=1, \ldots, n$. 
Remark 5.1. Denote $D=1-\sum_{j=1}^{n} \widetilde{u}_{0 j \mathcal{T}}$ and $D_{K}=1-\sum_{j=1}^{n} \widetilde{u}_{0 j K}, K \in \mathcal{T}_{0} . D$ satisfies

$$
\left\{\begin{array}{l}
\sum_{\sigma \in \mathcal{E}_{K}} F_{K, \sigma}=0, \forall K \in \mathcal{T}_{0} \\
F_{K, \sigma}=\frac{m(\sigma)}{d_{\sigma}}\left(D_{K}-D_{L}\right), \forall \sigma \in \mathcal{E}_{i n t}, \text { if } \sigma=\sigma_{K / L} \\
F_{K, \sigma}=\frac{m(\sigma)}{d_{\sigma}} D_{K}, \text { if } \sigma \subset\left(\cup_{j=1}^{n} \gamma_{j}^{\prime}\right), \sigma \in \mathcal{E}_{K}, \quad \text { and } 0 \text { else }
\end{array}\right.
$$

Let us take the sum for $K \in \mathcal{T}_{0}$ and reorder the summation over the set of edges, that gives

$$
\sum_{K \in \mathcal{T}_{0}} \sum_{\sigma \in \mathcal{E}_{K}} F_{K, \sigma} D_{K}=0=\sum_{\sigma=\sigma_{K / L} \in \mathcal{E}_{i n t}} \frac{m(\sigma)}{d_{\sigma}}\left(D_{K}-D_{L}\right)^{2}+\sum_{\sigma \subset\left(\cup_{j=1}^{n} \gamma_{j}^{\prime}\right)} \frac{m(\sigma)}{d_{\sigma}} D_{K}^{2}
$$

So, $D_{K}=0, \forall K \in \mathcal{T}_{0}$, that is to say $\sum_{j=1}^{n} \widetilde{u}_{0 j \mathcal{T}}=1$.

Now consider problem $\widetilde{P}_{j}, j=1, \ldots, n$ and consider a numerical approximation $\widetilde{u}_{j \mathcal{T}}$ of $\widetilde{u}_{j}$. Integrate the first equation $\triangle \widetilde{u}_{j}=0$ over each control volume $K \in \mathcal{T}_{j}$ and approximate the fluxes by difference quotients as above. Denote by $\widetilde{u}_{j K}$ an approximation of $\widetilde{u}_{j}\left(x_{K}\right), K \in \mathcal{T}_{j}$. The finite volume scheme is given below.

$$
(S j D 2)\left\{\begin{aligned}
& \sum_{\sigma \in \mathcal{E}_{K}} F_{K, \sigma}=0, \forall K \in \mathcal{T}_{j} \\
& F_{K, \sigma}= \frac{m(\sigma)}{d_{\sigma}}\left(\widetilde{u}_{j L}-\widetilde{u}_{j K}\right), \forall \sigma \in \mathcal{E}_{i n t}, \text { if } \sigma=\sigma_{K / L} \\
& F_{K, \sigma}=\frac{m(\sigma)}{d_{\sigma}}\left(1-\widetilde{u}_{j K}\right), \forall \sigma \subset \gamma_{j}^{\prime \prime}, \sigma \in \mathcal{E}_{K} \\
&=\frac{m(\sigma)}{d_{\sigma}}\left(-\widetilde{u}_{j K}\right), \forall \sigma \subset \hat{\beta}_{j}^{\varepsilon}, \sigma \in \mathcal{E}_{K} \\
&=0, \quad \forall \sigma \subset \partial \Omega(j) \backslash\left(\gamma_{j}^{\prime \prime} \cup \hat{\beta}_{j}^{\varepsilon}\right), \sigma \in \mathcal{E}_{K}
\end{aligned}\right.
$$

Define $\widetilde{u}_{j} \mathcal{T}(x, y)=\widetilde{u}_{j K},(x, y) \in K, K \in \mathcal{T}_{j}, j=1, \ldots, n$.

We study the error estimates for $\widetilde{P}_{0 j}$ and $\widetilde{P}_{j}, j=1, \ldots, n$, in section 6 .

5.3. Numerical approximation of $u^{d}$. We consider an approximate value of $u^{d}$, solution of $(2.2)$ defined by $u_{\mathcal{T}}^{d}(x, y)=\left\{\begin{array}{l}u_{j} \mathcal{T}(x, y),(x, y) \in \Omega(j), j=0, \ldots, n \\ v_{j} \mathcal{T}\left(x^{e_{j}}\right),(x, y) \in B_{j}^{\varepsilon}, x^{e_{j}} \in\left(\delta, l_{j}-\delta\right), j=1, \ldots, n\end{array}\right.$ with

$$
\left\{\begin{array}{l}
v_{j \mathcal{T}}=\bar{v}_{j \mathcal{T}}+\beta_{j \mathcal{T}} \widetilde{v}_{j 0}+\alpha_{j \mathcal{T}}, j=1, \ldots, n \\
u_{0 \mathcal{T}}=\sum_{j=1}^{n-1}\left(\left(\alpha_{j \mathcal{T}}+\beta_{j \mathcal{T}}\right)-\left(\alpha_{n \mathcal{T}}+\beta_{n \mathcal{T}}\right)\right) \widetilde{u}_{0 j \mathcal{T}}+\alpha_{n \mathcal{T}}+\beta_{n \mathcal{T}} \\
u_{j \mathcal{T}}=\alpha_{j \mathcal{T}} \widetilde{u}_{j \mathcal{T}}, j=1, \ldots, n
\end{array}\right.
$$


where $\left(\beta_{1 \mathcal{T}}, \ldots, \beta_{n \mathcal{T}}, \alpha_{1 \mathcal{T}}, \ldots, \alpha_{n \mathcal{T}}\right)$ is solution of the system

$(5.9)\left(S_{\alpha}\right)\left\{\begin{array}{l}\frac{1}{\theta_{j} \varepsilon} \sum_{k=1}^{n-1}\left(\left(\alpha_{k} \mathcal{T}+\beta_{k \mathcal{T}}\right)-\left(\alpha_{n \mathcal{T}}+\beta_{n \mathcal{T}}\right)\right) \sum_{\substack{\sigma \in \mathcal{E}_{K} \\ \sigma \subset \gamma_{j}^{\prime}}} \frac{m(\sigma)}{d_{\sigma}}\left(\delta_{j k}-\widetilde{u}_{0 k K}\right) \\ -\frac{\beta_{j} \mathcal{T}}{2 \delta-l_{j}}=\frac{\bar{v}_{j, 1}}{h_{1 / 2}^{e_{j}}, j=1, \ldots, n-1} \\ \sum_{j=1}^{n}-\frac{\theta_{j}}{\theta_{n}} \frac{\beta_{j \mathcal{T}}}{2 \delta-l_{j}}=\sum_{j=1}^{n} \frac{\theta_{j}}{\theta_{n}} \frac{\bar{v}_{j, 1}}{h_{1 / 2}^{e_{j}}} \\ \frac{1}{\theta_{j} \varepsilon} \alpha_{j \mathcal{T}} \sum_{\substack{\sigma \in \mathcal{E}_{K} \\ \sigma \subset \gamma_{j}^{\prime \prime}}} \frac{m(\sigma)}{d_{\sigma}}\left(1-\widetilde{u}_{j K}\right)+\frac{\beta_{j \mathcal{T}}}{2 \delta-l_{j}}=\frac{\bar{v}_{j, N_{j}}}{h_{N_{j}+1 / 2}^{e_{j}}}, j=1, \ldots, n\end{array}\right.$

Let us prove that $\left(S_{\alpha}\right)$ has a single solution.

We first write the system in a more symmetric way using $\widetilde{u}_{0 n \mathcal{T}}=1-\sum_{j=1}^{n-1} \widetilde{u}_{0 j} \mathcal{T}$ (Remark 5.1).

So that $\left.u_{0 \mathcal{T}}=\sum_{j=1}^{n}\left(\alpha_{j \mathcal{T}}+\beta_{j \mathcal{T}}\right)\right) \widetilde{u}_{0 j \mathcal{T}}$.

It is easy to see that $u_{0} \mathcal{T}$ is solution of

$$
\left\{\begin{array}{c}
\sum_{\sigma \in \mathcal{E}_{K}} F_{K, \sigma}=0, \forall K \in \mathcal{T}_{0} \\
F_{K, \sigma}=\frac{m(\sigma)}{d_{\sigma}} \sum_{j=1}^{n}\left(\alpha_{j \mathcal{T}}+\beta_{j \mathcal{T}}\right)\left(\widetilde{u}_{0 j L}-\widetilde{u}_{0 j K}\right), \forall \sigma \in \mathcal{E}_{i n t}, \text { if } \sigma=\sigma_{K / L} \\
F_{K, \sigma}=\frac{m(\sigma)}{d_{\sigma}} \sum_{j=1}^{n}\left(\alpha_{j \mathcal{T}}+\beta_{j \mathcal{T}}\right)\left(\delta_{j k}-\widetilde{u}_{0 j K}\right),, \forall \sigma \subset \gamma_{k}^{\prime}, \sigma \in \mathcal{E}_{K} \\
=0, \quad \forall \sigma \subset \partial \Omega(0) \backslash\left(\cup_{j=1}^{n} \gamma_{j}^{\prime}\right), \sigma \in \mathcal{E}_{K}
\end{array}\right.
$$

Using the above expression of $F_{K, \sigma}$ in $\sum_{K \in \mathcal{T}_{0}} \sum_{\sigma \in \mathcal{E}_{K}} F_{K, \sigma}=0$ we get

$$
\sum_{j=1}^{n} \sum_{k=1}^{n}\left(\alpha_{k \mathcal{T}}+\beta_{k \mathcal{T}}\right) \sum_{\substack{\sigma \in \mathcal{E}_{K} \\ \sigma \subset \gamma_{j}^{\prime}}} \frac{m(\sigma)}{d_{\sigma}}\left(\delta_{j k}-\widetilde{u}_{0 k K}\right)=0
$$

This yields the system $\left(S_{\alpha}^{\prime}\right)$ below, equivalent to $\left(S_{\alpha}\right)$. 
$(5.11)\left(S^{\prime} \alpha\right)\left\{\begin{array}{l}\sum_{k=1}^{n}\left(\alpha_{k \mathcal{T}}+\beta_{k \mathcal{T}}\right) \sum_{\substack{\sigma \in \mathcal{E}_{K} \\ \sigma \subset \gamma_{j}^{\prime}}} \frac{m(\sigma)}{d_{\sigma}}\left(\delta_{j k}-\widetilde{u}_{0 k K}\right)-\frac{\beta_{j} \mathcal{T} \theta_{j} \varepsilon}{2 \delta-l_{j}} \\ =\frac{\bar{v}_{j, 1}}{h_{1 / 2}^{e_{j}}} \theta_{j} \varepsilon, j=1, \ldots, n \\ \alpha_{j \mathcal{T}} \sum_{\substack{\sigma \in \mathcal{E}_{K} \\ \sigma \subset \gamma_{j}^{\prime \prime}}} \frac{m(\sigma)}{d_{\sigma}}\left(1-\widetilde{u}_{j K}\right)+\frac{\beta_{j \mathcal{T}} \theta_{j} \varepsilon}{2 \delta-l_{j}}=\frac{\bar{v}_{j, N_{j}}}{h_{N_{j}+1 / 2}^{e_{j}}} \theta_{j} \varepsilon, j=1, \ldots, n\end{array}\right.$

Denote $\left.u_{0 K}=\sum_{j=1}^{n}\left(\alpha_{j \mathcal{T}}+\beta_{j \mathcal{T}}\right)\right) \widetilde{u}_{0 j K}, K \in \mathcal{T}_{0}$

Using the expression (5.10) for $F_{K, \sigma}$ and taking into account that

$\sum_{K \in \mathcal{T}_{0}} \sum_{\sigma \in \mathcal{E}_{K}} F_{K, \sigma} u_{0 K}=0$

$\left.=-\sum_{\sigma=\sigma_{K / L} \in \mathcal{E}_{i n t}} \frac{m(\sigma)}{d_{\sigma}}\left(u_{0 L}-u_{0 K}\right)^{2}+\sum_{j=1}^{n} \sum_{\sigma \subset \gamma_{j}^{\prime}} \frac{m(\sigma)}{d_{\sigma}}\left(\left(\alpha_{j} \mathcal{T}+\beta_{j} \mathcal{T}\right)-u_{0 K}\right)\right) u_{0 K}$

We deduce

$$
\left.\sum_{j=1}^{n} \sum_{\sigma \subset \gamma_{j}^{\prime}} \frac{m(\sigma)}{d_{\sigma}}\left(\left(\alpha_{j} \mathcal{T}+\beta_{j \mathcal{T}}\right)-u_{0 K}\right)\right)\left(\alpha_{j} \mathcal{T}+\beta_{j} \mathcal{T}\right) \geq 0
$$

Then applying the maximum principle for solution $\widetilde{u}_{j \mathcal{T}}(5.7)$ to problem $(\mathrm{SjD} 2)$ we prove that $\forall j=1, \ldots, n, \forall K \in \mathcal{T}_{0}, 0 \leq \widetilde{u}_{j K} \leq 1$. This yields

$$
\sum_{\sigma \in \mathcal{E}_{K}} \frac{m(\sigma)}{d_{\sigma}}\left(1-\widetilde{u}_{j K}\right) \geq 0, j=1, \ldots, n
$$

Mention that $\left(S^{\prime}{ }_{\alpha}\right)$ is a linear system of the form $A_{\mathcal{T}}^{\prime} U_{\mathcal{T}}=B_{\mathcal{T}}^{\prime}$ with $U_{\mathcal{T}}^{T}=\left(\beta_{1 \mathcal{T}}, \ldots, \beta_{n \mathcal{T}}, \alpha_{1 \mathcal{T}}, \ldots, \alpha_{n \mathcal{T}}\right)$. $\left(S_{\alpha}\right)$ has a unique solution if and only if $\left(S^{\prime}{ }_{\alpha}\right)$ has a unique solution, i.e. if and only if $A_{\mathcal{T}}^{\prime} U_{\mathcal{T}}=0 \Rightarrow U_{\mathcal{T}}=0$. We set again $T=\left[\begin{array}{cc}I_{n} & I_{n} \\ 0 & I_{n}\end{array}\right]$.

$A_{\mathcal{T}}^{\prime} U_{\mathcal{T}}=0 \Rightarrow\left(T U_{\mathcal{T}}\right)^{T} A_{\mathcal{T}}^{\prime} U_{\mathcal{T}}=0$

$\Rightarrow \sum_{j=1}^{n}\left(\alpha_{j \mathcal{T}}+\beta_{j \mathcal{T}}\right) \sum_{k=1}^{n}\left(\alpha_{k \mathcal{T}}+\beta_{k} \mathcal{T}\right) \sum_{\substack{\sigma \in \mathcal{E}_{K} \\ \sigma \subset \gamma^{\prime}}} \frac{m(\sigma)}{d_{\sigma}}\left(\delta_{j k}-\widetilde{u}_{0 k K}\right)$

$-\sum_{j=1}^{n}\left(\alpha_{j \mathcal{T}}+\beta_{j} \mathcal{T}\right) \frac{\theta_{j} \varepsilon \beta_{j} \mathcal{T}}{2 \delta-l_{j}}+\sum_{j=1}^{n} \alpha_{j \mathcal{T}}^{2} \sum_{\substack{\sigma \in \mathcal{E}_{K} \\ \sigma \subset \gamma_{j}^{\prime \prime}}} \frac{m(\sigma)}{d_{\sigma}}\left(1-\widetilde{u}_{j K}\right)$

$$
+\sum_{j=1}^{n} \frac{\theta_{j} \varepsilon \alpha_{j \mathcal{T}} \beta_{j \mathcal{T}}}{2 \delta-l_{j}}=0
$$

Estimates (5.12) and (5.13) prove that $\left(T U_{\mathcal{T}}\right)^{T} A_{\mathcal{T}}^{\prime} U_{\mathcal{T}}$ is a sum of non-negative terms, and so, all these terms are equal to zero: $\alpha_{j \mathcal{T}}=\beta_{j \mathcal{T}}=0, j=1, \ldots, n$, that is to say $U_{\mathcal{T}}=0$. This proves that $\left(S_{\alpha}\right)$ has a unique solution. 
5.4. Hybrid equivalent numerical scheme for the solution of the partially decomposed problem. In the previous sections we have constructed the numerical finite volume scheme for every auxiliary problem $(\mathrm{SjD} 1), \mathrm{SjD} 2),(\mathrm{S} 0 \mathrm{jD} 2)$ and $\left(S_{\alpha}\right)$ and then constructed the approximate solution according to formula (3.1), while one can discretize directly the partially decomposed problem (2.2). Such discretization will be constructed in the present section. It is equivalent to the separate and independent discretization of the auxiliary problems and the combination of their solution by (3.1).

Introduce the following notations for the values of $u_{j \mathcal{T}}$ and $v_{j \mathcal{T}}$ :

$$
\left\{\begin{array}{l}
u_{j \mathcal{T}}(x, y)=u_{j K},(x, y) \in K, K \in \mathcal{T}_{j}, j=0, \ldots, n \\
v_{j \mathcal{T}}\left(x^{e_{j}}\right)=v_{j i}, x^{e_{j}} \in\left(x_{i-1 / 2}^{e_{j}}, x_{i+1 / 2}^{e_{j}}\right), i=1, \ldots, N_{j}, j=1, \ldots, n
\end{array}\right.
$$

Here $u_{j K}$ is an approximation of $u_{j}\left(x_{K}\right), K \in \mathcal{T}_{j}, j=0, \ldots, n$; and $v_{j i}$ is an approximation of $v_{j}\left(x_{i}\right), i=0, \ldots, N_{j}+1, j=1, \ldots, n$, satisfying

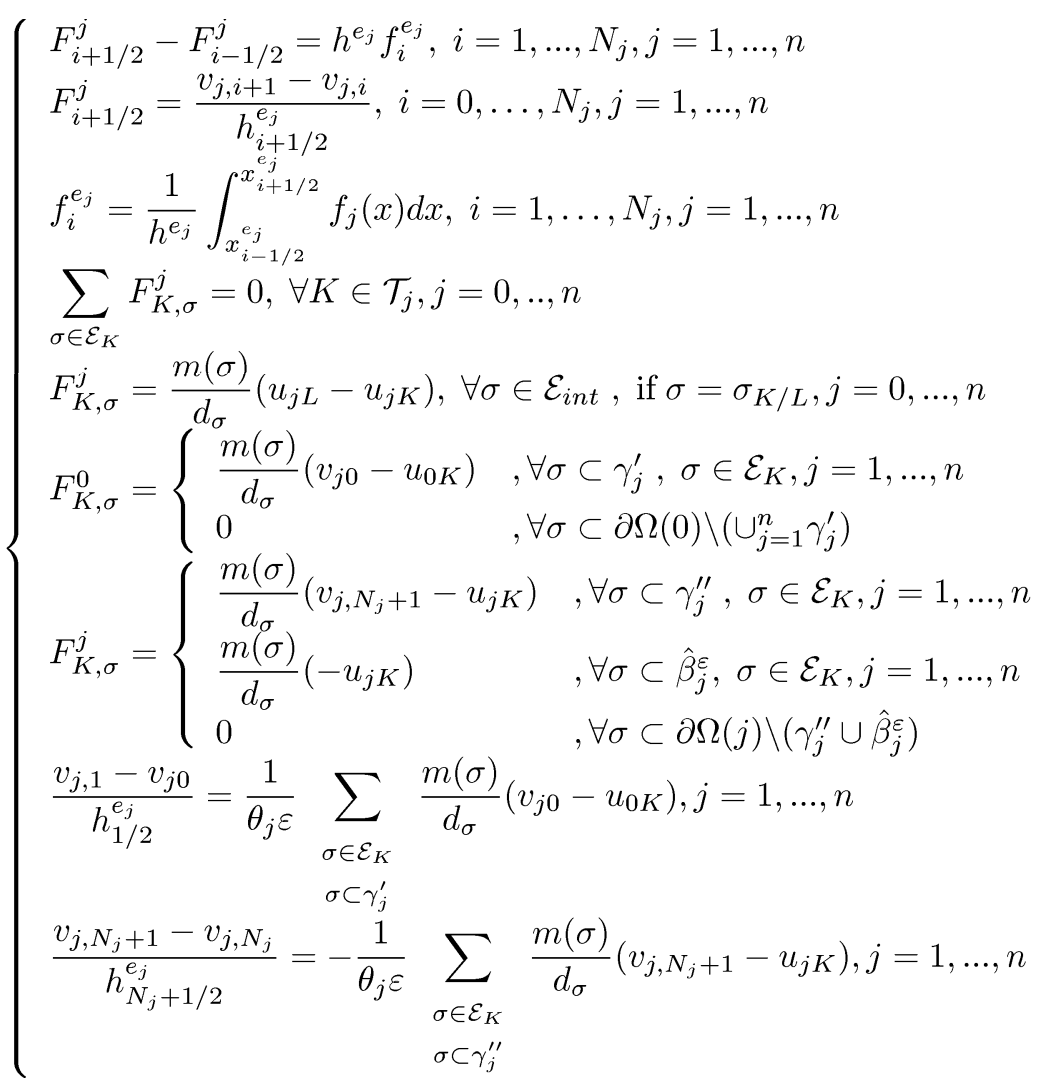

Mention that this numerical scheme (5.14) is equivalent to the scheme constructed in the previous sections, where the solution has been compiled with help of the auxiliary problems, i.e., 


$$
\left\{\begin{array}{l}
v_{j i}=\bar{v}_{j i}+\alpha_{0 j} \mathcal{T} \widetilde{v}_{j 0}\left(x_{i}^{e_{j}}\right)+\alpha_{j} \mathcal{T} \widetilde{v}_{j j}\left(x_{i}^{e_{j}}\right)=\bar{v}_{j i}+\beta_{j \mathcal{T}} \widetilde{v}_{j 0}\left(x_{i}^{e_{j}}\right)+\alpha_{j \mathcal{T}} \\
i=0, \ldots, N_{j}+1, j=1, \ldots, n \\
u_{0 K}=\sum_{j=1}^{n} \alpha_{0 j \mathcal{T}} \widetilde{u}_{0 j K}=\sum_{j=1}^{n-1}\left(\alpha_{0 j \mathcal{T}}-\alpha_{0 n \mathcal{T}}\right) \widetilde{u}_{0 j K}+\alpha_{0 n \mathcal{T}} \\
u_{j K}=\alpha_{j \mathcal{T}} \widetilde{u}_{j K}, K \in \mathcal{T}_{j}, j=1, \ldots, n
\end{array}\right.
$$

where $\alpha_{0, j} \mathcal{T}=\alpha_{j \mathcal{T}}+\beta_{j} \mathcal{T}, \mathrm{j}=1, \ldots, \mathrm{n}$.

6. Error estimate. The goal of the section is an error estimate for $\| u^{d}-$ $u_{\mathcal{T}}^{d} \|_{L^{2}\left(\Omega_{\varepsilon}\right)}$. Let us express this norm in the following way

$$
\left\|u^{d}-u_{\mathcal{T}}^{d}\right\|_{L^{2}\left(\Omega_{\varepsilon}\right)}^{2}=\sum_{j=0}^{n}\left\|u_{j}-u_{j \mathcal{T}}\right\|_{L^{2}(\Omega(j))}^{2}+\varepsilon \sum_{j=1}^{n} \theta_{j}\left\|v_{j}-v_{j \mathcal{T}}\right\|_{L^{2}\left(\delta, l_{j}-\delta\right)}^{2}
$$

and get an estimate for it using (3.5) (or (3.1) ) and (5.8). To this end let us evaluate $\left\|\bar{v}_{j}-\bar{v}_{j \mathcal{T}}\right\|_{L^{2}\left(\delta, l_{j}-\delta\right)},\left\|\widetilde{u}_{j}-\widetilde{u}_{j} \mathcal{T}\right\|_{L^{2}(\Omega(j))},\left\|\widetilde{u}_{0 j}-\widetilde{u}_{0 j \mathcal{T}}\right\|_{L^{2}(\Omega(0))}$; then $\left|\alpha_{j}-\alpha_{j \mathcal{T}}\right|$, and finally, $\left|\beta_{j}-\beta_{j \mathcal{T}}\right|$, i.e. $\left|\alpha_{0, j}-\alpha_{0, j \mathcal{T}}\right|, \mathbf{j}=1, \ldots, \mathrm{n}$.

Denote $h=\max \left(h_{j}, j=0, \ldots, n ; h^{e_{j}}, j=1, \ldots, n\right)$.

Assume that $\frac{h \ln \varepsilon}{\varepsilon} \rightarrow 0$ as $\varepsilon \rightarrow 0$ and $h \rightarrow 0$, i.e. that

$$
h=o\left(\frac{\varepsilon}{\ln \varepsilon}\right), \quad(\varepsilon \rightarrow 0) .
$$

6.1. Error estimate for (S0jD2) and (SjD2). Consider problems $\widetilde{P}_{0 j}$ and $\widetilde{P}_{j}, j=1, \ldots, n$.

Apply the discrete maximum principle, satisfied by the scheme, in order to perform the error estimate (see [15]). It gives $\left\|\widetilde{u}_{0 j \mathcal{T}}\right\|_{L^{\infty}(\Omega(0))} \leq 1, j=1, \ldots, n$, and, $\left\|\widetilde{u}_{j} \mathcal{T}\right\|_{L^{\infty}(\Omega(j))} \leq 1, j=1, \ldots, n$. We deduce that

$$
\left\|\widetilde{u}_{0 j \mathcal{T}}\right\|_{L^{2}(\Omega(0))}=O(\sqrt{\varepsilon \delta}), \quad\left\|\widetilde{u}_{j} \mathcal{T}\right\|_{L^{2}(\Omega(j))}=O(\sqrt{\varepsilon \delta}), \quad j=1, \ldots, n
$$

First, let us obtain an error estimate (as in [19]) for ( $\mathrm{SjD} 2)$. Define the spaces $H=\left\{v \in H^{1}(\Omega(j)),\left.v\right|_{\gamma_{j}^{\prime \prime} \cup \hat{\beta}_{j}^{\varepsilon}}=0\right\}, j=1, \ldots, n$, and the associated discrete norms.

For any piecewise constant fonction $W$ defined on $\Omega(j)$ such that $W(x, y)=W_{K},(x, y) \in$ $K, K \in \mathcal{T}_{j}$, denote $\|W\|_{L^{2}(\Omega(j))}=\left(\sum_{K \in \mathcal{T}} m(K) W_{K}^{2}\right)^{1 / 2}$ and

$$
\|U\|_{H, \mathcal{T}}=\left(\sum_{\sigma=\sigma_{K / L} \in \mathcal{E}_{i n t}} \frac{m(\sigma)}{d_{\sigma}}\left(W_{L}-W_{K}\right)^{2}+\sum_{\substack{\sigma \in \mathcal{E}_{K} \\ \sigma \subset \gamma_{j}^{\prime \prime} \cup \hat{\beta}_{j}^{\varepsilon}}} \frac{m(\sigma)}{d_{\sigma}} W_{K}^{2}\right)^{1 / 2}
$$


From the asymptotic analysis of $\widetilde{P}_{j}$, we see that $\left\|\nabla^{2} \widetilde{u}_{j}\right\|_{L^{\infty}(\Omega(j))}=O\left(\frac{1}{\varepsilon \delta}\right)$. The consistency error of the flux is defined by

$$
R_{K, \sigma}=\frac{1}{m(\sigma)}\left(\int_{\sigma} \frac{\partial \widetilde{u}_{j}}{\partial n} d \gamma-F_{K, \sigma}^{*}\right)
$$

for all $\sigma \in \mathcal{E}_{K}$ and for all $K \in \mathcal{T}_{j}$. Here $F_{K, \sigma}^{*}$ is the expression obtained from (5.7) replacing $\widetilde{u}_{j K}$ by $\widetilde{u}_{j}\left(x_{K}\right)$.

So, the order of $R_{K, \sigma}$ is $h_{j} \times\left\|\nabla^{2} \widetilde{u}_{j}\right\|_{L^{\infty}(\Omega(j))}$, and so $\left|R_{K, \sigma}\right|=O\left(\frac{h_{j}}{\varepsilon \delta}\right)$.

Writing the flux balance for any $K \in \mathcal{T}_{j}$, we get :

$$
\sum_{\sigma \in \mathcal{E}_{K}} \int_{\sigma} \frac{\partial \widetilde{u}_{j}}{\partial n} d \gamma-\sum_{\sigma \in \mathcal{E}_{K}} F_{K, \sigma}=0
$$

Define $e_{j} \mathcal{T}$, a function from $\Omega(j)$ to $\mathbb{R}$, constant over each control volume : $e_{j} \mathcal{T}(x, y)=$ $e_{j K},(x, y) \in K$, with $e_{j K}=\widetilde{u}_{j}\left(x_{K}\right)-\widetilde{u}_{j K}$, for all $K \in \mathcal{T}_{j}$.

Then one can write (6.4) in terms of $R_{K \sigma}$ and $e_{j K}$. Multiply this last equation by $e_{j K}$. Taking the sum of all equations for $K \in \mathcal{T}_{j}$, reorder the summation over the set of edges and use the Cauchy-Schwarz-Bunyakovskii inequality. This yields $\left\|e_{j \mathcal{T}}\right\|_{H, \mathcal{T}}=O\left(\frac{h_{j}}{\sqrt{\varepsilon \delta}}\right)$. Then applying the discrete version of the Poincaré inequality [15] we get $\left\|e_{j} \mathcal{T}\right\|_{L^{2}\left(\Omega_{j}\right)}=O\left(h_{j}\right)$.

To conclude, let us write $\widetilde{u}_{j}-\widetilde{u}_{j \mathcal{T}}$ in terms of $e_{j} \mathcal{T}$. The bound of the first derivatives of $\widetilde{u}_{j}$ is $O\left(\frac{1}{\delta}\right)$ and so,

$$
\left\|\widetilde{u}_{j}-\widetilde{u}_{j \mathcal{T}}\right\|_{L^{2}\left(\Omega_{j}\right)}=O\left(h_{j}\right), j=1, \ldots, n
$$

Now, we are going to obtain an error estimate for (S0jD2) in the same manner. For any piecewise constant function $W$ defined on $\Omega(0)$ and such that $W(x, y)=$ $W_{K},(x, y) \in K, K \in \mathcal{T}_{0}$, denote

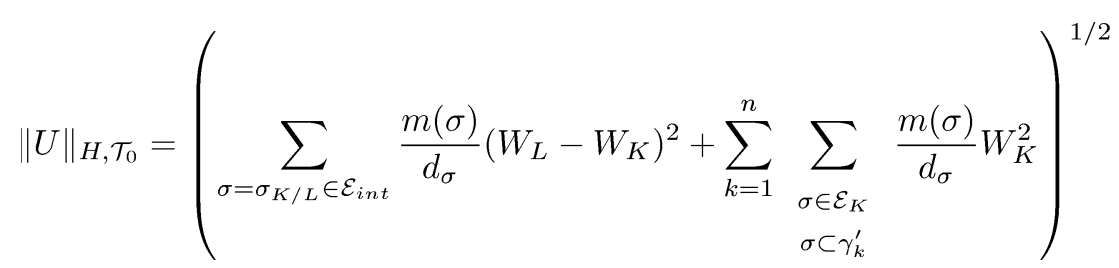

Define as above $e_{0 j} \mathcal{T}$, the function from $\Omega(0)$ to $\mathbb{R}$, constant over each control volume $: e_{0 j \mathcal{T}}(x, y)=e_{0 j K},(x, y) \in K$, with $e_{0 j K}=\widetilde{u}_{0 j}\left(x_{K}\right)-\widetilde{u}_{0 j K}$, for all $K \in \mathcal{T}_{0}$.

As above, we prove that $\left\|e_{0 j \mathcal{T}}\right\|_{H, \mathcal{T}_{0}}=O\left(\frac{h_{0}}{\sqrt{\varepsilon \delta}}\right)$. Applyig again the discrete version of the Poincaré inequality we get $\left\|e_{0 j \mathcal{T}}\right\|_{L^{2}(\Omega(j))}=O\left(h_{0}\right)$, and so,

$$
\left\|\widetilde{u}_{0 j}-\widetilde{u}_{0 j} \mathcal{T}\right\|_{L^{2}(\Omega(0))}=O\left(h_{0}\right), j=1, \ldots, n
$$


6.2. Estimate for $\left|\alpha_{j}-\alpha_{j \mathcal{T}}\right|$ and $\left|\beta_{j}-\beta_{j \mathcal{T}}\right|, \mathbf{j}=\mathbf{1}, \ldots, \mathbf{n}$. Consider system (3.8) rewritten in the form of the system $A U=B$, where $A, B$ are the block matrices defined in Section 4.3 and $U^{T}=\left(\beta_{1}, \ldots, \beta_{n}, \alpha_{1}, \ldots, \alpha_{n}\right)$. This system is approximated by $(5.9)\left(S_{\alpha}\right)$ :

$$
A_{\mathcal{T}} U_{\mathcal{T}}=B_{\mathcal{T}}
$$

where $U_{\mathcal{T}}^{T}=\left(\beta_{1 \mathcal{T}}, \ldots, \beta_{n \mathcal{T}}, \alpha_{1 \mathcal{T}}, \ldots, \alpha_{n \mathcal{T}}\right)$. Let us evaluate $\left\|U-U_{\mathcal{T}}\right\|$. In Section 4.3 matrix $A$ was presented in the form $A=\left[\begin{array}{cc}M & C \\ D & I_{n}\end{array}\right]$, and $B=\left[\begin{array}{c}B 1 \\ B 2\end{array}\right]$. Here we do the same with respect to $A_{\mathcal{T}}$ and $B_{\mathcal{T}}$; they are the block matrices defined by $A_{\mathcal{T}}=\left[\begin{array}{cc}M_{\mathcal{T}} & C_{\mathcal{T}} \\ D & G_{\mathcal{T}}\end{array}\right]$, and $B_{\mathcal{T}}=\left[\begin{array}{l}B 1_{\mathcal{T}} \\ B 2_{\mathcal{T}}\end{array}\right]$. The matrices $M_{\mathcal{T}}, C_{\mathcal{T}}, D, G_{\mathcal{T}}$ have dimension $n \times n$ and the vectors $B 1_{\mathcal{T}}, B 2_{\mathcal{T}}$ have dimension $n \times 1$.

Define $M_{\mathcal{T}}=\left(M_{\mathcal{T} j k}\right)_{1 \leq j, k \leq n}$, where

$$
\begin{aligned}
M_{\mathcal{T} j k} & =\frac{m_{j k}}{r_{j j}}-\frac{\delta_{j k}}{\left(2 \delta-l_{j}\right) r_{j j}} \text { if } j, k=1, \ldots, n-1 \\
& =-\sum_{i=1}^{n-1} \frac{m_{j i}}{r_{j j}} \text { if } j=1, \ldots, n-1, k=n \\
& =-\frac{\theta_{k}}{\theta_{n}} \frac{1}{\left(2 \delta-l_{k}\right)} \text { if } j=n, k=1, \ldots, n, \\
m_{j k}=\frac{1}{\theta_{j} \varepsilon} & \sum_{\sigma \in \mathcal{E}_{K}} \frac{m(\sigma)}{d_{\sigma}}\left(\delta_{j k}-\widetilde{u}_{0 k K}\right), j, k=1, \ldots, n-1 ; \\
C_{\mathcal{T}}=\left(C_{\mathcal{T}_{j k}^{\prime}}\right)_{1 \leq j, k \leq n} & \text { with } \\
C_{\mathcal{T} j k} & =\frac{m_{j k}}{r_{j j}} \text { if } j, k=1, \ldots, n-1 \\
& =-\sum_{i=1}^{n-1} \frac{m_{j i}}{r_{j j}} \text { if } j=1, \ldots, n-1, k=n \\
& =0 \text { if } j=n, k=1, \ldots, n
\end{aligned}
$$

$G_{\mathcal{T}}=\left(G_{\mathcal{T} j k}\right)_{1 \leq i, k \leq n}$, where

$$
\begin{aligned}
G_{\mathcal{T}_{j k}} & =\frac{g_{j}}{t_{j}} \delta_{j k}, j, k=1, \ldots, n, \\
g_{j} & =\frac{1}{\theta_{j} \varepsilon} \sum_{\substack{\sigma \in \mathcal{E}_{K} \\
\sigma \subset \gamma_{j}^{\prime \prime}}} \frac{m(\sigma)}{d_{\sigma}}\left(1-\widetilde{u}_{j K}\right), j=1, \ldots, n ;
\end{aligned}
$$

$B 1_{\mathcal{T}}=\left(B 1_{\mathcal{T} j}\right)_{1 \leq j \leq n}$ and $B 2_{\mathcal{T}}=\left(B 2_{\mathcal{T} j}\right)_{1 \leq j \leq n}$, where

$$
B 1_{\mathcal{T}_{j}}=\frac{\bar{v}_{j, 1}}{h_{1 / 2}^{e_{j}} r_{j j}}, j=1, \ldots, n-1, \text { and } B 1_{n}=\sum_{i=1}^{n} \frac{\theta_{i}}{\theta_{n}} \frac{\bar{v}_{i, 1}}{h_{1 / 2}^{e_{i}}}
$$




$$
B 2_{\mathcal{T} j}=\frac{\bar{v}_{j, N_{j}}}{h_{N_{j}+1 / 2}^{e_{j}} t_{j}}, j=1, \ldots, n
$$

We have $A_{\mathcal{T}}\left(U_{\mathcal{T}}-U\right)+\left(A_{\mathcal{T}}-A\right) U=B_{\mathcal{T}}-B$.

Let us mention that $A_{\mathcal{T}}-A=\left[\begin{array}{cc}M_{\mathcal{T}}-M & C_{\mathcal{T}}-C \\ 0 & G_{\mathcal{T}}-I_{n}\end{array}\right]$, with $M_{\mathcal{T}}-M=C_{\mathcal{T}}-C$ (the $n^{\text {th }}$ line is always zero). Let us evaluate the order of size of $\left\|M_{\mathcal{T}}-M\right\|$ and $\left\|G_{\mathcal{T}}-I_{n}\right\|$. We have

$$
\begin{aligned}
M_{\mathcal{T} j k}-M_{j k} & =\frac{m_{j k}-r_{j k}}{r_{j j}} \text { if } j, k=1, \ldots, n-1 \\
& =-\sum_{i=1}^{n-1} \frac{m_{j i}-r_{j i}}{r_{j j}} \text { if } j=1, \ldots, n-1, k=n \\
& =0 \text { if } j=n, k=1, \ldots, n \\
G_{\mathcal{T} j k} & -I_{n j k}=\frac{g_{j}-t_{j}}{t_{j}} \delta_{j k}, j, k=1, \ldots, n,
\end{aligned}
$$

These two quantities are of the same order. Let us study for instance just the order of the second one.

$$
\begin{aligned}
& g_{j}-t_{j}=\frac{1}{\theta_{j} \varepsilon}\left(\sum_{\substack{\sigma \in \mathcal{E}_{K} \\
\sigma \subset \gamma_{j}^{\prime \prime}}} \frac{m(\sigma)}{d_{\sigma}}\left(1-\widetilde{u}_{j K}\right)-\int_{\gamma_{j}^{\prime \prime}} \frac{\partial \widetilde{u}_{j}}{\partial n} d \gamma\right) \\
& =\frac{1}{\theta_{j} \varepsilon} \sum_{\sigma \in \mathcal{E}_{K}}\left(\frac{m(\sigma)}{d_{\sigma}}\left(1-\widetilde{u}_{j K}\right)-\int_{\sigma} \frac{\partial \widetilde{u}_{j}}{\partial n} d \gamma\right) \\
& =\frac{1}{\theta_{j} \varepsilon} \sum_{\sigma \in \mathcal{E}_{K}}^{\sigma \subset \gamma_{j}^{\prime \prime}}\left(\frac{m(\sigma)}{d_{\sigma}}\left(1-\widetilde{u}_{j}\left(x_{K}\right)\right)-\int_{\sigma} \frac{\partial \widetilde{u}_{j}}{\partial n} d \gamma\right)+\frac{1}{\theta_{j} \varepsilon} \sum_{\sigma \in \mathcal{E}_{K}} \frac{m(\sigma)}{d_{\sigma}} e_{j K} \\
& \sigma \subset \gamma_{j}^{\prime \prime} \quad \sigma \subset \gamma_{j}^{\prime \prime} \\
& =\frac{1}{\theta_{j} \varepsilon} \sum_{\sigma \in \mathcal{E}_{K}}\left(-m(\sigma) R_{K \sigma}\right)+\frac{1}{\theta_{j} \varepsilon} \sum_{\sigma \in \mathcal{E}_{K}} \frac{m(\sigma)}{d_{\sigma}} e_{j K} \\
& \sigma \subset \gamma_{j}^{\prime \prime} \quad \sigma \subset \gamma_{j}^{\prime \prime}
\end{aligned}
$$

where $R_{K \sigma}$ has been defined in (6.3) and $e_{j K}$ just few lines below (6.3). So the first term of the sum is $O\left(\frac{h_{j}}{\varepsilon \delta}\right)$. Let us study the second term. The Cauchy-SchwarzBunyakovskii inequality and (5.4) yield

$\left|\frac{1}{\theta_{j} \varepsilon} \sum_{\substack{\sigma \in \mathcal{E}_{K} \\ \sigma \subset \gamma_{j}^{\prime \prime}}} \frac{m(\sigma)}{d_{\sigma}} e_{j K}\right| \leq \frac{1}{\theta_{j} \varepsilon}\left\|e_{j} \mathcal{T}\right\|_{H, \mathcal{T}}\left(\sum_{\sigma \subset \gamma_{j}^{\prime \prime}} \frac{m(\sigma)}{d_{\sigma}}\right)^{1 / 2} \leq \sqrt{\frac{1}{\zeta h_{j} \theta_{j} \varepsilon}}\left\|e_{j \mathcal{T}}\right\|_{H, \mathcal{T}}$ 
We have seen in Section 6.1 that $\left\|e_{j \mathcal{T}}\right\|_{H, \mathcal{T}}=O\left(\frac{h_{j}}{\sqrt{\varepsilon \delta}}\right)$. So the second term of the sum is of order $O\left(\frac{1}{\varepsilon} \sqrt{\frac{h_{j}}{\delta}}\right)$.

Since $\frac{1}{t_{j}}=O(\delta)$, we have $\left|G_{\mathcal{T} j k}-I_{n j k}\right|=O\left(\frac{h_{j}}{\varepsilon}\right)+O\left(\frac{\sqrt{h_{j} \delta}}{\varepsilon}\right)$, and in the same way $\left|M_{\mathcal{T} j k}-M_{j k}\right|=O\left(\frac{h_{0}}{\varepsilon}\right)+O\left(\frac{\sqrt{h_{0} \delta}}{\varepsilon}\right)$.

We conclude from above that $\left\|M_{\mathcal{T}}-M\right\|$ and $\left\|G_{\mathcal{T}}-I_{n}\right\|$ are $O\left(\frac{h}{\varepsilon}\right)+O\left(\frac{\sqrt{h \delta}}{\varepsilon}\right)$.

Denote $\alpha_{\mathcal{T}}^{T}=\left(\alpha_{1 \mathcal{T}}, \ldots, \alpha_{n} \mathcal{T}\right)$ and $\beta_{\mathcal{T}}^{T}=\left(\beta_{1 \mathcal{T}}, \ldots, \beta_{n \mathcal{T}}\right)$. We have

$\begin{aligned} & A_{\mathcal{T}}\left(U_{\mathcal{T}}-U\right)+\left(A_{\mathcal{T}}-A\right) U=B_{\mathcal{T}}-B \\ \Leftrightarrow & \left\{\begin{array}{l}M_{\mathcal{T}}\left(\beta_{\mathcal{T}}-\beta\right)+C_{\mathcal{T}}\left(\alpha_{\mathcal{T}}-\alpha\right)=B 1_{\mathcal{T}}-B 1-\left(M_{\mathcal{T}}-M\right)(\alpha+\beta)=R 1 \\ D\left(\beta_{\mathcal{T}}-\beta\right)+G_{\mathcal{T}}\left(\alpha_{\mathcal{T}}-\alpha\right)=B 2_{\mathcal{T}}-B 2-\left(G_{\mathcal{T}}-I_{n}\right) \alpha=R 2,\end{array}\right.\end{aligned}$

where $R 1$ and $R 2$ are the right hand sides. $G_{\mathcal{T}}$ is a regular matrix. Indeed, $G_{\mathcal{T}}=$ $I_{n}+G_{\mathcal{T}}-I_{n}$ and due to condition (6.1) $\frac{h}{\varepsilon}$ and $\frac{\sqrt{h \delta}}{\varepsilon}$ tend to zero, and so $\left\|G_{\mathcal{T}}-I_{n}\right\|<1$ as $h, \varepsilon$ tend to zero.

Let us write $G_{\mathcal{T}}-I_{n}$ in the form $G_{\mathcal{T}}-I_{n}=\frac{h}{\varepsilon} A_{2}+\frac{\sqrt{h \delta}}{\varepsilon} A_{3}$, where the matrices $A_{2}$ and $A_{3}$ are $O(1)$. So $G_{\mathcal{T}}^{-1}=\sum_{k=0}^{\infty}(-1)^{k}\left(\frac{h}{\varepsilon} A_{2}+\frac{\sqrt{h \delta}}{\varepsilon} A_{3}\right)^{k}=I_{n}+R$, where $R$ is a matrix satisfying $\|R\|=O\left(\frac{h}{\varepsilon}\right)+O\left(\frac{\sqrt{h \delta}}{\varepsilon}\right)$. Of course, $\left\|G_{\mathcal{T}}^{-1}\right\|=O(1)$.

The previous system can be so written in the form

$$
\left\{\begin{array}{l}
\left(M_{\mathcal{T}}-C_{\mathcal{T}} G_{\mathcal{T}}^{-1} D\right)\left(\beta_{\mathcal{T}}-\beta\right)=R 1-C_{\mathcal{T}} G_{\mathcal{T}}^{-1} R 2 \\
\alpha_{\mathcal{T}}-\alpha=G_{\mathcal{T}}^{-1} R 2-G_{\mathcal{T}}^{-1} D\left(\beta_{\mathcal{T}}-\beta\right)
\end{array}\right.
$$

Consider now $R 1$ and $R 2$. We have, for instance, for $\mathrm{j}=1, \ldots, \mathrm{n}-1$

$$
\begin{gathered}
r_{j j}\left|\left(B 1_{\mathcal{T}}-B 1\right)_{j}\right|=\left|\bar{v}_{j}^{\prime}(\delta)-\frac{\bar{v}_{j, 1}}{h_{1 / 2}^{e_{j}}}\right| \\
\leq\left|\bar{v}_{j}^{\prime}(\delta)-\frac{\bar{v}_{j}\left(x_{1}^{e_{j}}\right)-\bar{v}_{j}(\delta)}{h_{1 / 2}^{e_{j}}}\right|+\frac{\left|\bar{e}_{j 1}\right|}{h_{1 / 2}^{e_{j}}}=O(\sqrt{h})
\end{gathered}
$$

This proves that $\left|\left(B 1_{\mathcal{T}}-B 1\right)_{j}\right|=O(\delta \sqrt{h}), \mathbf{j}=1, \ldots, \mathrm{n}-1$. But $\left|\left(B 1_{\mathcal{T}}-B 1\right)_{n}\right|=O(\sqrt{h})$. So that $\left\|B 1_{\mathcal{T}}-B 1\right\|=O(\sqrt{h})$. In the same manner, we obtain $\left\|B 2_{\mathcal{T}}-B 2\right\|=O(\delta \sqrt{h})$. We deduce: $R 1=O(\sqrt{h})+O\left(\frac{h}{\varepsilon}\right)+O\left(\frac{\sqrt{h \delta}}{\varepsilon}\right)$ and $R 2=O(\delta \sqrt{h})+O\left(\frac{h \delta}{\varepsilon}\right)+$ $O\left(\delta \frac{\sqrt{h \delta}}{\varepsilon}\right)$

Now, let us return to equation (6.14). Matrix $M_{\mathcal{T}}-C_{\mathcal{T}} G_{\mathcal{T}}^{-1} D$ is regular. Indeed, we have 
$M_{\mathcal{T}}-C_{\mathcal{T}} G_{\mathcal{T}}^{-1} D=\left(M_{\mathcal{T}}-M\right)+M-\left(C_{\mathcal{T}}-C+C\right) G_{\mathcal{T}}^{-1} D$ $=M-C D+\left(M_{\mathcal{T}}-M\right)-\left(C_{\mathcal{T}}-C\right) G_{\mathcal{T}}^{-1} D-C R D=A_{0}+\delta A_{1}+\frac{h}{\varepsilon} A_{4}+\frac{\sqrt{h \delta}}{\varepsilon} A_{5}$, where $A_{4}$ and $A_{5}$ are $O(1)$, and $A_{0}, A_{1}$ have been defined in (4.15) and (4.16). Since $A_{0}$ is regular, with $(6.1)$, we conclude that $M_{\mathcal{T}}-C_{\mathcal{T}} G_{\mathcal{T}}^{-1} D$ is regular. And so, $\left(M_{\mathcal{T}}-C_{\mathcal{T}} G_{\mathcal{T}}^{-1} D\right)^{-1}=\left(\sum_{k=0}^{\infty}(-1)^{k}\left(\delta A_{0}^{-1} A_{1}+\frac{h}{\varepsilon} A_{0}^{-1} A_{4}+\frac{\sqrt{h \delta}}{\varepsilon} A_{0}^{-1} A_{5}\right)^{k}\right) A_{0}^{-1}=$ $A_{0}^{-1}+V$

where $V$ is a matrix such that $\|V\|=O(\delta)+O\left(\frac{h}{\varepsilon}\right)+O\left(\frac{\sqrt{h \delta}}{\varepsilon}\right)$. This yields that $\beta_{\mathcal{T}}-\beta=\left(A_{0}^{-1}+V\right)\left(R 1-C_{\mathcal{T}} G_{\mathcal{T}}^{-1} R 2\right)$ has the same order than $R 1$ so that

$$
\left\|\beta_{\mathcal{T}}-\beta\right\|=O(\sqrt{h})+O\left(\frac{h}{\varepsilon}\right)+O\left(\frac{\sqrt{h \delta}}{\varepsilon}\right)=O\left(\frac{\sqrt{h \delta}}{\varepsilon}\right)
$$

and we conclude

$$
\left\|\alpha_{\mathcal{T}}-\alpha\right\|=O(\delta \sqrt{h})+O\left(\frac{h \delta}{\varepsilon}\right)+O\left(\delta \frac{\sqrt{h \delta}}{\varepsilon}\right)=O\left(\delta \frac{\sqrt{h \delta}}{\varepsilon}\right)
$$

6.3. Error estimate : the main result. Consider once more the norm

$$
\left\|u^{d}-u_{\mathcal{T}}^{d}\right\|_{L^{2}\left(\Omega_{\varepsilon}\right)}^{2}=\sum_{j=0}^{n}\left\|u_{j}-u_{j \mathcal{T}}\right\|_{L^{2}(\Omega(j))}^{2}+\varepsilon \sum_{j=1}^{n} \theta_{j}\left\|v_{j}-v_{j \mathcal{T}}\right\|_{L^{2}\left(\delta, l_{j}-\delta\right)}^{2}
$$

Consider $\alpha_{0, j}$ and $\alpha_{0, j} \mathcal{T}, j=1, \ldots, n$. We see that $\left|\alpha_{0, j}-\alpha_{0 j \mathcal{T}}\right|=\left|\alpha_{j}+\beta_{j}-\alpha_{j \mathcal{T}}-\beta_{j \mathcal{T}}\right|=$ $O\left(\frac{\sqrt{h \delta}}{\varepsilon}\right)$. So, $\left\|u_{0}-u_{0 \mathcal{T}}\right\|_{L^{2}(\Omega(0))} \leq \sum_{j=1}^{n}\left|\alpha_{0 j}\right|\left\|\widetilde{u}_{0 j}-\widetilde{u}_{0 j \mathcal{T}}\right\|_{L^{2}(\Omega(0))}+\sum_{j=1}^{n} \mid \alpha_{0 j}-$ $\alpha_{0 j \mathcal{T}} \mid\left\|\widetilde{u}_{0 j \mathcal{T}}\right\|_{L^{2}(\Omega(0))}$

and $\left\|u_{0}-u_{0 \mathcal{T}}\right\|_{L^{2}(\Omega(0))}=O\left(\sqrt{\frac{h}{\varepsilon}} \delta\right)$.

Then, for $j=1, \ldots, n$, we have

$\left\|u_{j}-u_{j \mathcal{T}}\right\|_{L^{2}(\Omega(j))} \leq\left|\alpha_{j}\right|\left\|\widetilde{u}_{j}-\widetilde{u}_{j \mathcal{T}}\right\|_{L^{2}(\Omega(j))}+\left|\alpha_{j}-\alpha_{j \mathcal{T}}\right|\left\|\widetilde{u}_{j \mathcal{T}}\right\|_{L^{2}(\Omega(j))}$.

So, we deduce that $\left\|u_{j}-u_{j \mathcal{T}}\right\|_{L^{2}(\Omega(j))}=O\left(\sqrt{\frac{h}{\varepsilon}} \delta^{2}\right)$.

Then, for $\mathrm{j}=1, \ldots, \mathrm{n}$, we have

$\left\|v_{j}-v_{j \mathcal{T}}\right\|_{L^{2}\left(\delta, l_{j}-\delta\right)} \leq\left\|\bar{v}_{j}-\bar{v}_{j \mathcal{T}}\right\|_{L^{2}\left(\delta, l_{j}-\delta\right)}+\left|\beta_{j}-\beta_{j \mathcal{T}}\right|\left\|\widetilde{v}_{j 0}\right\|_{L^{2}\left(\delta, l_{j}-\delta\right)}+\left|\alpha_{j}-\alpha_{j \mathcal{T}}\right|$ and we deduce that $\left\|v_{j}-v_{j \mathcal{T}}\right\|_{L^{2}\left(\delta, l_{j}-\delta\right)}=O\left(\frac{\sqrt{h \delta}}{\varepsilon}\right)$.

Finally, we have

$$
\begin{aligned}
& \left\|u^{d}-u_{\mathcal{T}}^{d}\right\|_{L^{2}\left(\Omega_{\varepsilon}\right)}^{2}=O\left(\frac{h}{\varepsilon} \delta^{2}\right)+\varepsilon O\left(\frac{h \delta}{\varepsilon^{2}}\right)=O\left(\frac{h \delta}{\varepsilon}\right) . \\
& \text { i.e., } \\
& \left\|u^{d}-u_{\mathcal{T}}^{d}\right\|_{L^{2}\left(\Omega_{\varepsilon}\right)}=O\left(\sqrt{\frac{h \delta}{\varepsilon}}\right) .
\end{aligned}
$$


So we have the following

THEOREM 6.1. Let $K$ be a positive number. There exists $\hat{K}$ independent of $\varepsilon$ and $h$, such that, if $\delta=\hat{K} \varepsilon|\ln (\varepsilon)|$ and $\frac{h|\ln (\varepsilon)|}{\varepsilon}$ tends to zero, then the approximate finite volume solution $u_{\mathcal{T}}^{d}$ of the partially decomposed problem converges to the solution of the Poisson equation $u_{\varepsilon}$ set in a thin finite rod structure $\Omega_{\varepsilon}$, as the mesh step $h$ and $\varepsilon$ tend to zero. The estimate holds:

$$
\left\|u_{\varepsilon}-u_{\mathcal{T}}^{d}\right\|_{L^{2}\left(\Omega_{\varepsilon}\right)}=O\left(\sqrt{\frac{h \delta}{\varepsilon}}\right)+O\left(\varepsilon^{K}\right)
$$

Remark 6.1. $\left\|u_{\varepsilon}-u_{\mathcal{T}}^{d}\right\|_{L^{2}\left(\Omega_{\varepsilon}\right)}=O(\sqrt{\varepsilon})$ because $\sqrt{\frac{h \delta}{\varepsilon}}=O\left(\sqrt{\frac{h|\ln (\varepsilon)|}{\varepsilon}} \sqrt{\varepsilon}\right)$ and $\frac{h|\ln (\varepsilon)|}{\varepsilon}$ tends to zero.

7. Numerical experiment. Consider the domain

$$
\begin{gathered}
\Omega_{\varepsilon}=(0,1) \times\left(-\frac{\varepsilon}{2}, \frac{\varepsilon}{2}\right) \cup\left(-\frac{\varepsilon}{2}, \frac{\varepsilon}{2}\right) \times(0,1) \cup\left\{x^{2}+y^{2}<\frac{\varepsilon^{2}}{4}\right\} \\
\cup\left\{(x-\varepsilon)^{2}+(y-\varepsilon)^{2}>\frac{\varepsilon^{2}}{4} ; \frac{\varepsilon}{2}<x<\varepsilon, \frac{\varepsilon}{2}<y<\varepsilon\right\},
\end{gathered}
$$

represented in the Figure 7.1. It differs from the defined earlier because it is not smoothened in the neighborhoods of the points $O_{1}=(1,0)$ and $O_{2}=(0,1)$. It mens that the domains $\omega_{1}$ and $\omega_{2}$ are removed from $\Omega_{\varepsilon}$. Here according to the introduced notations, $n=2, \theta_{1}=\theta_{2}=1, l_{1}=l_{2}=1, \hat{\beta}_{1}=\left\{x=1, y \in\left(-\frac{\varepsilon}{2}, \frac{\varepsilon}{2}\right)\right\}$, and $\hat{\beta}_{2}=\left\{y=1, x \in\left(-\frac{\varepsilon}{2}, \frac{\varepsilon}{2}\right)\right\}$.

Consider problem (2.1) set in this domain with the right hand side

$$
f(x, y)= \begin{cases}1080(3 x-2) & \text { if } x \geq 0.5 \\ 1080(3 y-2) & \text { if } y \geq 0.5 \\ 0 & \text { else }\end{cases}
$$

In this case the exact solution is known and is equal to

$$
u_{\epsilon}(x, y)= \begin{cases}540\left(x^{3}-2 x^{2}\right)+135(5 x-1) & \text { if } x \geq 0.5 \\ 540\left(y^{3}-2 y^{2}\right)+135(5 y-1) & \text { if } y \geq 0.5 \\ 0 & \text { else }\end{cases}
$$

In the Figure 7.1, we represent the approximate solution $u_{\epsilon \mathcal{T}}$ of $u_{\varepsilon}$ obtained with the standard $2 \mathrm{D}$ finite volume scheme $[19]$ for the Laplace equation. The mesh is a classical admissible triangular mesh, the size of the mesh is $h=0.025$.

The partially decomposed problem is the associated 1D-2D hybrid problem set in the domain obtained by the truncation of $\Omega_{\varepsilon}$ at $x=\delta$ and $y=\delta$. We choose $\varepsilon=\delta=0.2$. Compare the results obtained by application of the $1 \mathrm{D}-2 \mathrm{D}$ corresponding finite volume scheme which gives an approximation for $u^{d}$, with the exact solution 

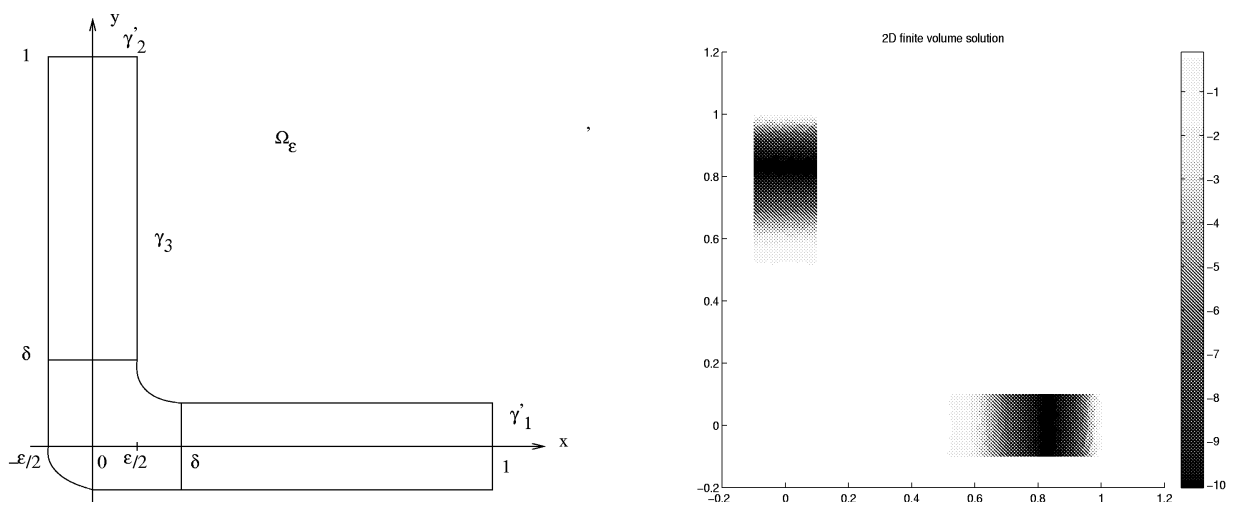

FIG. 7.1. The initial domain and finite volume solution with $h=0.025$
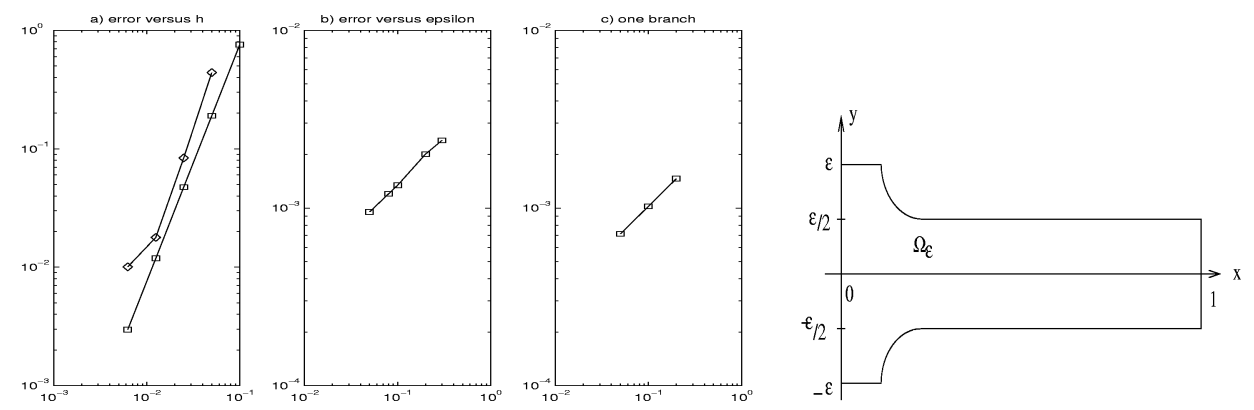

FIG. 7.2. Error between the 1D-2D approximate solution and the exact solution a) as a function of $h b$ ) as a function of $\varepsilon c$ ) and d) special case one branch

of (2.1). This last scheme is constructed with the same admissible mesh as above for the 2D-region $(x \leq \delta, y \leq \delta)$, and by using a regular 1D-finite volume scheme with a $1 \mathrm{D}$-mesh of the same size when $x>\delta$ or $y>\delta$. Denote $\mathcal{T}$ the described $1 \mathrm{D}-2 \mathrm{D}$ mesh. The 1D-2D approximation is denoted as usual $u_{\mathcal{T}}^{d}$, where $u_{\mathcal{T}}^{d}=\sum_{K \in \mathcal{T}} u_{\mathcal{T} K}^{d} \mathbf{1}_{K}$ and $u_{\mathcal{T} K}^{d}=u_{K}$ if $K$ is in the 2D-region, and $u_{\mathcal{T} K}^{d}=v_{j i}, j \in\{1,2\}$, if $i$ is such that $x_{K} \in I_{i}^{e_{j}}$. Denote $u_{\mathcal{T}}^{e}=\sum_{K \in \mathcal{T}} u_{\varepsilon}\left(x_{K}\right) \mathbf{1}_{K}$.

In the figure $7.2 \mathrm{a}$ ) (curve marked with squares), we give the error $\left\|u_{\mathcal{T}}^{d}-u_{\mathcal{T}}^{e}\right\|_{L^{2}\left(\Omega_{\varepsilon}\right)}$ as a function of the mesh size $h$, for a family of admissible meshes. The mesh step $h$ is taken equal to 0.1 (coarse grid), 0.05, 0.025, 0.0125 and finally 0.00625 (fine grid). The slope of the line gives the order of convergence versus $h$ of the 1D-2D scheme (logarithmic scale). The order is slightly below $h^{2}$ (the slope is 1.9996) in $L^{2}$ norm. This superconvergence is classically observed for finite volume schemes in $2 \mathrm{D}$ for the Laplace equation [4]. We observe the same order of convergence for the 1D-2D hybrid finite volume scheme.

The numerical order of convergence is often better than the theoretical one. For finite volume schemes in $2 \mathrm{D}$, with an admissible mesh, for the Laplace equation, a first-order convergence is proved [19]. The observed numerical superconvergence (see the figure 7.2 a) may come from the regularity of the solution. To have more elements to answer this question, we solve the problem (2.1) associated to another functions as the right hand side $f$. In these cases, the exact solution being unknown, we compare 
the approximate solution computed with a size of the mesh $h$ with the approximate one computed with a size of the mesh $h / 2$. Doing so, of course, we obtain again a second order convergence if $f$ is given by (7.1). But we no longer observe superconvergence, for instance, for the following test cases, (respectively cases 2,3 , and 4):

$$
\begin{aligned}
& f(x, y)= \begin{cases}10^{5} \sin (x-0.3)(x-0.7)^{2} & \text { if } 0.3 \leq x \geq 0.7 \\
10^{5} \sin (y-0.5)(y-0.9)^{2} & \text { if } 0.5 \leq x \geq 0.9 \\
0 & \text { else }\end{cases} \\
& f(x, y)= \begin{cases}10^{5} \sin (x-0.2)(x-0.7)^{4} & \text { if } 0.2 \leq x \geq 0.7 \\
10^{5} \sin (y-0.5)(y-0.9)^{4} & \text { if } 0.5 \leq x \geq 0.9 \\
0 & \text { else }\end{cases} \\
& f(x, y)= \begin{cases}10^{6} \sin (x-0.2)(x-0.7)^{7} & \text { if } 0.2 \leq x \geq 0.7 \\
10^{6} \sin (y-0.5)(y-0.9)^{7} & \text { if } 0.5 \leq x \geq 0.9 \\
0 & \text { else }\end{cases}
\end{aligned}
$$

In the figure 7.3 , on the right, we show the convergence order obtained in these four cases (the first case is (7.1)). The dashed line stands for the case $1, \diamond$ for the case $2, *$ case 3 and + case 4 . We give in the table 7.1 the slopes step by step. We conclude, according to the examples, the order may be just one. This result is in better adequation with the theoretical prevision for the convergence rate.

\begin{tabular}{|c|c|c|c|c|}
\hline$h / 2$ and $h$ & case1 & case2 & case3 & case4 \\
\hline 0.05 and 0.1 & - & - & - & - \\
\hline 0.025 and 0.05 & 2.0846 & 1.9965 & 1.9578 & 1.7906 \\
\hline 0.0125 and 0.025 & 2.0204 & 1.6515 & 1.0236 & 1.4873 \\
\hline 0.00625 and 0.0125 & 2.0120 & 1.2633 & 1.3428 & 2.5784 \\
\hline \multicolumn{5}{|c}{ TABLF 7.1}
\end{tabular}

Convergence rate of the $1 D$-2D finite volume scheme in four cases.

In the figure $7.2 \mathrm{~b}$ ), the error $\left\|u_{\mathcal{T}}^{d}-u_{\mathcal{T}}^{e}\right\|_{L^{2}\left(\Omega_{\varepsilon}\right)}$ is presented for a family of admissible meshes of the same mesh size $h=0.005$ as a function of $\varepsilon$ ( $\varepsilon$ takes the values : $0.05,0.08,0.1,0.2,0.3)$. Some shapes of domain $\Omega_{\varepsilon}$ are shown on the figure 7.3. Here, the 1D-2D scheme approximates the associated 1D-2D hybrid problem set in the domain obtained by the truncation of $\Omega_{\varepsilon}$ with $\delta=\varepsilon|\ln (\varepsilon)|$. As above the slope of the line in the Figure $7.2 \mathrm{~b}$ ) gives the order of convergence of the 1D-2D scheme versus $\varepsilon$. The order of convergence is $\varepsilon^{0.5287}$ in the $L^{2}$ norm, and it corresponds to the theoretical result.

In the Figure $7.2 \mathrm{c}$ ) and d), the order of convergence of the 1D-2D scheme versus $\varepsilon$ is presented in the case of one branch (a channel-like domain $\Omega_{\varepsilon}$, see $[15 \mid$ ). The order of convergence is 0.5170 in the $L^{2}$ norm and it corresponds to the theoretical prediction of order $O\left(\sqrt{\frac{h}{\varepsilon}} \delta\right)$, proved in [15]. We observe that the error is of the same order independently of the number of branchs of $\Omega_{\varepsilon}$. However, this observation is not yet confirmed theoretically. 

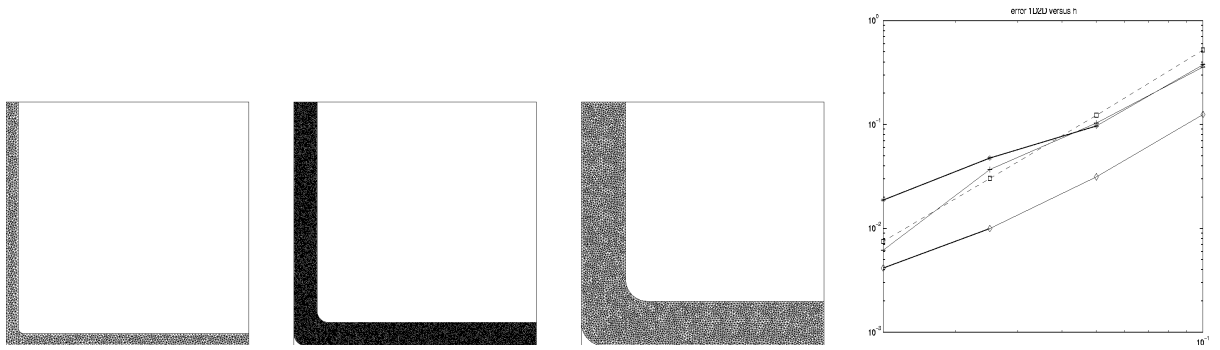

FIG. 7.3. Domain $\Omega_{\varepsilon}$ with $\varepsilon=0.05,0.1,0.2$. Convergence order for tests case $2 \diamond, 3 *, 4+$.

\begin{tabular}{|c|c|c|c|c|c|c|}
\hline$\varepsilon$ & 0.2 & 0.2 & 0.2 & 0.2 & 0.05 & 0.2 \\
$\delta$ & 0.2 & 0.2 & 0.2 & 0.2 & 0.15 & 0.32 \\
$h$ & 0.1 & 0.025 & 0.0125 & 0.00625 & 0.005 & 0.005 \\
\hline$L^{2}$-norm & 0.1653 & 0.0151 & 0.0099 & 0.0042 & 0.0043 & 0.0013 \\
\hline
\end{tabular}

Relative error in $L^{2}$ norm between the $2 D$ and $1 D$-2D finite volume schemes

At last, we compare the results obtained with the 2D standard finite volume scheme [19] and with the hybrid 1D-2D finite volume scheme.

At the figure $7.2 \mathrm{a}$ ), we notice that the error curves versus $h$ for the $2 \mathrm{D}$ standard finite volume scheme $(\diamond)$ and for the 1D-2D scheme (curve marked with squares) are almost parallel to a line of slope 2. Moreover, the figure shows that the precision is approximately 2 times better for the hybrid scheme than for the standard 2D scheme. We notice the four points obtained with the 2D-scheme are not in a line. Indeed, the fourth point obtained with $h=0.00625$ with the $2 \mathrm{D}$-scheme is not in a good position. This comes from numerical imprecision because the matrix is close to singular or badly scaled. On the contrary, there is no stability problem with the 1D-2D scheme, probably since the real $2 \mathrm{D}$ part of the numerical solution is limited.

Table 7.2 shows the relative error between the 2D approximate solution and the 1D-2D approximate solution in the $L^{2}$ norm. We see that the error between the 2D and $1 \mathrm{D}-2 \mathrm{D}$ finite volume schemes is very small (except for column 1 because the space step is very large, $h=0.1$ ). So the partial asymptotic domain decomposition is a very efficient method, and the proposed above hybrid finite volume scheme allows to obtain numerical results as accurate as those obtained with the standard finite volume scheme.

8. Conclusion. The previous result on the finite volume implementation of the MAPDD [15] is generalised for an arbitrary 2D rod structure. The numerical tests confirm the theoretical convergence rate.

This approach may be useful in the context of coupling the models of different dimension implemented in some codes : it gives theoretically justified interface conditions at the common boundaries.

Acknowledgments. The authors gratefully acknowledge the joint French-Russian PICS CNRS grant "Mathematical modelling of blood diseases" and the SFR MODMAD grant of the University of Saint-Etienne/ENISE, the second author gratefully 
acknowledges grant Number 14.740.11.0875 "Multiscale problems: analysis and methods" and grant Number 12.740.11.140 "Construction, analysis and application of methods for boundary value problems and initial-boundary value problems with multiple characteristic scales" of Russian Ministry of Education and Research.

\section{R.FFFR.FNCFS}

[1] S.Agmon, A.Douglis, L.Nirenberg. Estimates near the boundary for solutions of elliptic partial differential equations satisfying general boundary conditions. I, II. Comm. Pure Appl. Math., 12 (4): 623-723, 1959, 17(1): 35-92, 1964.

[2] N.S. BaKhVALOV. On optimization of the methods of solution of boundary value problems with boundary layers. Zh.Vyc.Mat.i Mat.Fis.(ZVMMF),9(4):841-859, 1969.

[3] H. BREzis, Analyse fonctionnelle: Théorie et Applications, Masson, 1983.

[4] K. Domelevo, And P. Omnes. A finite volume method for the Laplace equation on almost arbitrary two-dimensional grids. M2AN, 39 (6) : 1203-1249, 2005.

[5] L. C. Evans. Partial differential equations. Graduate Studies in Mathematics, American Mathematical Society, volume 19.

[6] F. Fontvieille, G. Panasenko and J. Pousin. FEM implementation for the asymptotic partial decomposition. Applicable Analysis, 86 (5) : 519-536, 2007.

[7] A.M.ILyin. Difference scheme for a differential equation with small parameter in the higher derivative. Mat. Zametki, 6(2),237-248, 1969.

[8] L.V.Kantorovich, V.I.Krylov. Approximate Methods of Higher Analysis, Noordhoff, Groningen, 1958.

[9] E. M. Landis, G. Panasenko. A theorem on the asymptotics of solutions of elliptic equations with coefficients periodic in all variables except one, Soviet Math Doklady, 1977, 18, 4, pp. 1140-1143.

[10] G.P. Panasenko. Asymptotic solutions of the elasticity theory system of equations for lattice and skeletal structures. Math.Sb.,183(1):89-113, 1992 (in Russian). English transl. by AMS in Russian Acad. Sci. Sbornik Math. 75(1):85-110, 1993.

[11] G.P. Panasenko. Method of asymptotic partial decomposition of domain. Mathematical Models and Methods in Applied Sciences, 8(1):139-156,1998.

[12] G.P. PANASENKo. Method of asymptotic partial decomposition of rod structures. International Journal of Computational, Civil and Structural Engineering (Begel House Publ.) 1(2):57-70, 2000 .

[13] G.P. Panasenko. Asymptotic expansion of the solution of Navier-Stokes equation in tube structure and partial asymptotic decomposition of the domain, Applicable Analysis an International Journal, 76(3-4) : 363-381, 2000.

[14] G.P. Panasenko. Multi-scale Modelling for Structures and Composites, Dordrecht etc.: Kluwer, 2005.

[15] G.P. Panasenko and M.-C. Viallon. The finite volume implementation of the partial asymptotic domain decomposition, Applicable Analysis an International Journal, 87(12) : 13971424,2008

[16] A. Quarteroni and L. Formaggia. Mathematical Modelling and Numerical Simulation of the Cardiovascular System. In Modelling of Living Systems, Handbook of Numerical Analysis Series. N. Ayache editor, 2002.

17] M.Vogelius. I.BABUSKA. On a dimensional reduction method. I.(The optimal selection of basis functions). Mathematics of Computations, 37(155):31-46, 1981; II.(Some approximationtheoretic results). Mathematics of Computations, 37(155):47-68, 1981; III. (A-posteriori error estimation and an adaptive approach). Mathematics of Computations, 37:361-384, 1981.

18] P. Arminjon, M-C Viallon, A. Madrane, A finite volume extension of the Lax-Friedrichs and Nessyahu-Tadmor schemes for conservation laws on unstructured grids, Int. Jour. of Comp. Fluid Dynamics, 9 (1), 1997, p. 1-22.

[19] R. Eymard, T. Gallouet, R. Herbin, Finite Volume methods, Handbook of Numerical Analysis. Vol VII, 2000, p. 713-1020, Editors : P.G. Ciarlet and J.L. Lions.

20] S.V. Patankar (1980), Numerical Heat Transfer and Fluid Flow, Series in Computational Methods in Mechanics and Thermal Sciences, Mikowycz and Sparrow Eds. (Mc Graw Hill)

[21] L. SaAs, I. Faille, F. Nataf and F. Willien, Domain decomposition for a finite volume method on non-matching grids., C. R. Acad. Sci. Paris, Ser. I Math., 338 (2004), 407-412.

$[22]$ J. Droniou, A recipe to couple two finite volume schemes for elliptic problems., Proceedings 
of Finite Volumes For Complex Applications V, ISTE, WILEY (2008).

23] P. Angot, F. Boyer And F. Hubert. Asymptotic and numerical modelling of flows in fractured porous media. M2AN, 43, 239-275, 2009.

24] D. CAILlerie, Thin elastic and periodic plates. Math.Methods Appl.Sci., 6(2):159-191, 1984.

25] P.G. Ciarlet, Plates and Junctions in Elastic Multi-structures. An Asymptotic Analysis. Paris: Masson, 1990.

26] P.G. Ciarlet, P. Destuynder , A justification of the two-dimensional plate model. J. Mécanique, 18:315-344, 1979.

27] A. L. GOLDENVEIZER, The principles of reducing three-dimensional problems of elasticity to two-dimensional problems of the theory of plates and shells. Proceedings, Eleventh International Congress of Theoretical and Applied Mechanics (H. Görtler, editor), pages 306-311. Berlin: Springer-Verlag, 1964.

[28] A.G.Kolpakov, Composite Structures Stressed, Springer, Berlin, Heidelberg, 2004, 229pp.

[29] V. A. Kozlov, V.G. Mazya And A.B. Movchan , Asymptotic Analysis of Fields in Multistructures. Oxford: Clarendon Press, 1999.

[30] M.V. Kozlova, AND G.P. PANASENKo, Averaging of the three-dimensional problem of elasticity theory for an inhomogeneous rod. Comput. Maths.Math.Phys., 31(10): 128-131, 1991.

[31] H. LE DRET , Modelling of the junction between two rods. J. Math. Pures Appl., 68:365-397, 1989.

[32] H. LE DRET, Problèmes Variationnels dans les Multi-domaines: Modélisation des Jonctions et Applications, RMA 19 , Masson, 1991.

[33] F. Murat, A. Sili, Comportement asymptotique des solutions du système de l' élasticité linéarisé anisotrope hétérogène dans des cylindres minces. Comptes Rendus Acad. Sci. Paris, Série I, 328(1) : 179-184, 1999.

[34] S.A. NAZAROV , Structure of solutions of elliptic boundary value problems in thin domains. Vestnik Leningrad University, 7:65-68, 1982.

[35] S.A NAzArov, Asymptotic Analysis of Thin Plates and Bars, Volume 1. Novosibirsk: Nauchnaya Kniga, 2002.(in Russian)

[36] G.P. PANASENKo, Boundary layer in homogenization problems for non - homogeneous media. S. K. Godunov, J. J. H. Miller, V. A. Novikov editors, Proceedings of the Fourth International Conference on Boundary and Interior Layers - Computational and Asymptotic Methods (BAIL 4), pages 398-402, Dublin:Boole Press , 1986

[37] G.P. Panasenko, Asymptotic analysis of bar systems.I. Russian Journal of Math. Physics, 2(3):325-352, 1994.

[38] G.P. Panasenko , Asymptotic analysis of bar systems.II. Russian Journal of Math. Physics, $4(1): 87-116,1996$.

[39] E. SANChEZ-PALENCIA, Passage à la limite de l'élasticité tri-dimensionnelle à la théorie asymptotique des coques minces. C.R. Acad. Sci. Paris, Sér. II, 311:909-916, 1990.

[40] L.Trabucho, J.M. Viaño, Derivation of generalized models for linear elastic beams by asymptotic expansion methods. P.G. Ciarlet and E. Sanchez-Palencia editors, Applications of Multiple Scalings in Mechanics, pages 302-315. Paris: Masson, 1987. 